\title{
MLFMA Analysis of Scattering from Multiple Targets In the Presence of a Half Space
}

\author{
Ling Li, Jiangqi He, Zhijun Liu and Lawrence Carin \\ Department of Electrical and Computer Engineering \\ Box 90291 \\ Duke University \\ Durham, NC 27708-0291
}

\begin{abstract}
The multi-level fast multipole algorithm (MLFMA) is applied to the analysis of planewave scattering from multiple conducting and/or dielectric targets, of arbitrary shape, situated in the presence of a dielectric half space. The multiple-target scattering problem is solved in an iterative fashion. In particular, the fields exciting each target are represented as the incident fields plus the scattered fields from all other targets. The scattered fields from each target are updated iteratively, until the induced currents on all targets have converged. The model is validated with an independent scattering algorithm, and example results are presented for several scenarios of interest to remote sensing.
\end{abstract}

\section{Introduction}

There are many remote-sensing applications for which one is interested in electromagnetic scattering from multiple targets in the presence of a half space. For example, there is significant interest in scattering from multiple trees, as well as in the detection of man-made targets situated in foliage. Until very recently such problems were beyond the means of available computational power, and approximate methods were required. For example, wave propagation in foliage has been modeled using an equivalent lossy dielectric layer situated above the earth [1,2]. Such a model has yielded remarkable accuracy and phenomenological understanding [1,2] at VHF frequencies, although the model is inappropriate when the wavelength is on the order of or smaller than the separation between trees.

More recently Tsang et al. [3] have considered scattering of electromagnetic fields from 
dielectric cylinders situated normal to the interface of a lossy dielectric half space. The size and relative positions of the cylinders are varied statistically, from which scattering statistics are obtained. This approach allows prediction of the scattering statistics from relatively realistic forests. To address the multi-target scattering problem, Tsang et al. [3] employ the following iterative procedure, for the case of multiple dielectric-cylinder targets. The excitation fields on a given target are represented as the initial incident fields, plus the scattered fields from all other targets. The scattering from each cylinder is analyzed independently, with the target-dependent excitation updated iteratively as the scattered fields from the targets are computed.

Motivated by the desire to consider a large number of cylinders, representative of a forest, the following approximation has been made in [3]. The scattered fields from a given cylinder are computed using the cylindrical-harmonic solution of an infinite-length dielectric cylinder in free space. The induced electric and magnetic currents on the infinite cylinder are then truncated to the dimensions of the cylinder of interest, with the scattered fields computed using the half-space Green's function. While this approximation allows consideration of large problems, it is of interest to consider more-realistic foliage models. The scattering of electromagnetic waves from trees has been investigated by several authors using numerous techniques [3-9]. In addition to the aforementioned infinite-cylinder model [3], authors have examined the use of volumetric electricfield integral equations, solved via an electric-dipole model [7]. This algorithm is applicable to tree models more realistic [7] than a simple finite-length cylinder.

While there have been numerous studies of electromagnetic scattering from tree-like problems, there has been little published work for the problem of a man-made target in the presence of trees. Even at VHF frequencies, many man-made targets are electrically large, mitigating the use of traditional models such as the method of moments (MoM) [10,11]. For the case of $N$ unknowns, an integral-equation solver such as MoM requires order $N^{2}$ memory, order $N^{2}$ computational complexity (CPU time) to fill the matrix, order $P \cdot N^{2} \mathrm{CPU}$ time to solve the resulting matrix equation via an iterative solver using $P$ iterations, or order $N^{3}$ CPU to solve the matrix via a direct solver [12]. For realistic problems such computational requirements are beyond the capacity of even modern computers. We have therefore developed a multi-level fast-multipole algorithm (MLFMA) for 
general conducting or dielectric targets in the presence of a half space [13-16]. Other researchers have developed related computational tools [17-21]. The MLFMA addresses an integral equation like MoM, but requires order $N \log N$ memory and $P \cdot N \log N$ CPU time, making tractable the analysis of scattering from previously unsolvable electromagnetic problems [13-21].

In the work presented here we extend the MLFMA to the analysis of multiple conducting and/or dielectric targets in the presence of a half space. We employ an iterative procedure analogous to that described above and developed by Tsang et al. [3]. In particular, the MLFMA is employed to rigorously solve for the scattered fields from a general conducting or dielectric target situated in isolation in the presence of a half space. The excitation fields on each target are the incident fields, plus the MLFMA-computed scattered fields from all other targets. This procedure has been employed for the related problem of scattering from body-of-revolution targets with arbitrary appendages [22]. The approach is naturally amenable to a parallel implementation, with each target analyzed on a separate processor, with the scattered fields from a given target communicated to all other targets. In this initial study we do not consider a parallel implementation, although we employ a careful use of computer memory such that the required memory is only that required of the largest target considered, rather than the cumulative memory required for all targets. This issue is discussed in greater detail below.

As indicated above, the analysis presented here is of interest for several remote-sensing problems. We first validate the iterative MLFMA solution with comparison to results computed via an independent scattering model. The canonical problem of scattering from two cylinders situated above a half space is also considered, this of interest in the future for validation of new models. We then present more-realistic results. In particular we consider scattering from two tree-like targets, a man-made vehicle situated behind a tree-like target, a buried target situated under a rough soil interface, and two unexploded ordnance (UXO) buried in soil.

The remainder of the paper is organized as follows. In Sec. II we present the iterative MLFMA formulation, with canonical test examples presented in Sec. III. Example scattering results of interest for remote-sensing problems are discussed in Sec. IV, with conclusions presented in Sec. 
V.

\section{Formulation}

\section{A. MLFMA analysis}

The multi-level fast-multipole algorithm (MLFMA) has been presented in several recent papers [13-21], and the interested reader is referred to these papers for an in-depth discussion of the formalism. We point out particular issues of interest for the problem considered here, with a focus on perfectly conducting targets for notational simplicity. We have also developed the MLFMA for dielectric targets [16], and in Secs. III and IV results are presented for multiple dielectric targets. The case of dielectric targets is analogous to that presented below, with additional notational and computational complexity [16]. For conducting targets we employ the combined-field formulation

$$
\begin{aligned}
& \boldsymbol{t} \cdot\left[\alpha \boldsymbol{E}^{i n c}(\boldsymbol{x})+\eta_{i}(1-\alpha) \boldsymbol{n} \times \boldsymbol{H}^{i n c}(\boldsymbol{x})\right]= \\
& \boldsymbol{t} \cdot\left[-\alpha \boldsymbol{E}^{s c a t}(\boldsymbol{x})+\eta_{i}(1-\alpha) \boldsymbol{J}(\boldsymbol{x})-\eta_{i}(1-\alpha) \boldsymbol{n} \times \boldsymbol{H}^{s c a t}(\boldsymbol{x})\right]
\end{aligned}
$$

with the scattered fields given by (inside half-space $i$ in which the target resides)

$$
\begin{gathered}
\boldsymbol{E}^{s c a t}(\boldsymbol{x})=-j \omega \mu_{i}\left[\boldsymbol{I}+\frac{\nabla \nabla}{k_{i}^{2}}\right] \cdot \int_{S^{\prime}} \boldsymbol{G}_{A i i}\left(\boldsymbol{x}, \boldsymbol{x}^{\prime}\right) \cdot \boldsymbol{J}\left(\boldsymbol{x}^{\prime}\right) d S^{\prime} \\
=-\underset{S^{\prime}}{j \omega \mu_{i i}} \boldsymbol{K}_{A i i}\left(\boldsymbol{x}, \boldsymbol{x}^{\prime}\right) \cdot \boldsymbol{J}\left(\boldsymbol{x}^{\prime}\right) d S^{\prime}+\frac{\nabla}{j \omega \epsilon_{i}} \int_{S^{\prime}} K_{\phi e}^{i i}\left(\boldsymbol{x}, \boldsymbol{x}^{\prime}\right) \nabla^{\prime} \cdot \boldsymbol{J}\left(\boldsymbol{x}^{\prime}\right) d S^{\prime} \\
\boldsymbol{H}^{\text {scat }}(\boldsymbol{x})=\nabla \times \int_{S^{\prime}} \boldsymbol{G}_{A i i}\left(\boldsymbol{x}, \boldsymbol{x}^{\prime}\right) \cdot \boldsymbol{J}\left(\boldsymbol{x}^{\prime}\right) d S^{\prime}
\end{gathered}
$$

The unit vectors $\boldsymbol{n}$ and $\boldsymbol{t}$ are normal and tangential to the scatterer surface, respectively; $\boldsymbol{x}$ is on the surface of the target; $\epsilon_{r}=\epsilon_{r i}{ }^{\prime}-j \sigma_{i} / \omega \epsilon_{o}, \mu_{i}$ and $k_{i}$ represent the complex relative permittivity, permeability and wavenumber of the region in which the target resides $\left(\sigma_{i}\right.$ is the associated conductivity and $\epsilon_{o}$ is the free-space permittivity); and $\omega$ is the angular frequency (an $\exp (j \omega t)$ time dependence is assumed and suppressed). Details on the half-space Green's function dyadics $\boldsymbol{G}_{A i i}$ and 
$\boldsymbol{K}_{A i i}$, as well as the scalar Green's function $K_{\phi e}{ }^{i i}$ have been given by Michalski and Zheng in [23], and we use their formulation $\mathrm{C}$.

The CFIE (1) includes the electric-field integral equation (EFIE, $\alpha=1$ ) [15], which must be employed for an open target, and the magnetic-field integral equation (MFIE, $\alpha=0)$ [15] as special cases. To avoid spurious interior resonances of the EFIE and MFIE solutions, we typically choose $\alpha$ in the range $\alpha=0.2$ to $\alpha=0.5$ if analyzing a closed perfectly conducting target.

To solve an integral equation of the form in (1), the unknown surface currents $\boldsymbol{J}(\boldsymbol{x})$ are expanded in a set of known functions with unknown coefficients, where here we employ the Rao, Wilton, Glisson (RWG) [24] basis functions. The integral equations are solved by using testing functions identical to the expansion functions, resulting in a matrix equation $\boldsymbol{Z} \boldsymbol{i}=\boldsymbol{v}$, where $\boldsymbol{Z}$ is an $N \times N$ impedance matrix, $\boldsymbol{i}$ is a $N \times 1$ column vector representing the unknown basis-function coefficients, and $v$ is an $N \times 1$ column vector representing the incident fields tested on the target surface. The fast multipole method (FMM) [25] utilizes properties of the free-space Green's function whereby interactions between expansion and testing functions are treated on a cluster-by-cluster basis, with each cluster defining a set of proximate basis functions. This approach is appropriate if the clusters are "far enough" apart, with this defined in a rigorous manner [13-21,25]. The remaining interactions, which are too close for the aforementioned technique, are treated as in the traditional MoM, this typically yielding a sparse and nearly diagonal matrix multiplication. In the MLFMA, multiple clusters sizes are considered consecutively, in a nested fashion, this yielding order $N \log$ $N$ memory requirements and order $P \cdot N \log N$ CPU time. The principal complication of interest here, in the context of the half-space problem, involves extending the FMM identities from the free-space case to the half-space problem. These issues are discussed in detail in [13-16].

\section{B. Multiple targets}

Assume that we have $M$ targets situated in region $i$ of a half-space problem. The formulation in (1) now generalizes to 


$$
\begin{aligned}
\boldsymbol{t}_{m} \cdot\left[\alpha_{m} \boldsymbol{E}^{i n c}\left(\boldsymbol{x}_{m}\right)\right. & \left.+\eta_{i}\left(1-\alpha_{m}\right) \boldsymbol{n}_{m} \times \boldsymbol{H}^{i n c}\left(\boldsymbol{x}_{m}\right)\right]= \\
& \eta_{i}\left(1-\alpha_{m}\right) \boldsymbol{t}_{m} \cdot \boldsymbol{J}\left(\boldsymbol{x}_{m}\right)+\boldsymbol{t}_{m} \cdot\left[-\alpha_{m} \boldsymbol{E}_{m}^{s c a t}\left(\boldsymbol{x}_{m}\right)-\eta_{i}\left(1-\alpha_{m}\right) \boldsymbol{n}_{m} \times \boldsymbol{H}_{m}^{s c a t}\left(\boldsymbol{x}_{m}\right)\right] \\
& +\boldsymbol{t}_{m} \cdot \sum_{k \neq m}\left[-\alpha_{m} \boldsymbol{E}_{k}^{s c a t}\left(\boldsymbol{x}_{m}\right)-\eta_{i}\left(1-\alpha_{m}\right) \boldsymbol{n}_{m} \times \boldsymbol{H}_{k}^{s c a t}\left(\boldsymbol{x}_{m}\right)\right]
\end{aligned}
$$

where we have an equation of the form in (3) for each of the $M$ targets. Note that in general the coefficient $\alpha_{m}$ is target dependent, since some targets may be open and others closed. The expressions $\boldsymbol{E}_{k}^{\text {scat }}\left(\boldsymbol{x}_{m}\right)$ and $\boldsymbol{H}_{k}^{\text {scat }}\left(\boldsymbol{x}_{m}\right)$ are the scattered electric and magnetic fields, respectively, observed on the surface of target $m$ (at $\boldsymbol{x}_{m}$ ) due to the $k$ th target. These expressions are given by

$$
\begin{aligned}
& \boldsymbol{E}_{k}^{s c a t}\left(\boldsymbol{x}_{m}\right)=-j \omega \mu_{i}\left[\boldsymbol{I}+\frac{\nabla \nabla}{k_{i}^{2}}\right] \cdot \int_{S_{k}^{\prime}} \boldsymbol{G}_{A i i}\left(\boldsymbol{x}_{m}, \boldsymbol{x}^{\prime}\right) \cdot \boldsymbol{J}\left(\boldsymbol{x}^{\prime}\right) d S^{\prime} \\
& =-j \omega \mu_{i} \int_{S_{k}^{\prime}} \boldsymbol{K}_{A i i}\left(\boldsymbol{x}_{m}, \boldsymbol{x}^{\prime}\right) \cdot \boldsymbol{J}\left(\boldsymbol{x}^{\prime}\right) d S^{\prime}+\frac{\nabla}{j \omega \epsilon_{i}} \int_{S_{k}^{\prime}} K_{\phi e}^{i i}\left(\boldsymbol{x}_{m}, \boldsymbol{x}^{\prime}\right) \nabla^{\prime} \cdot \boldsymbol{J}\left(\boldsymbol{x}^{\prime}\right) d S^{\prime} \\
& \boldsymbol{H}_{k}^{s c a t}\left(\boldsymbol{x}_{m}\right)=\nabla \times \underset{S_{k}^{\prime}}{ } \boldsymbol{G}_{A i i}\left(\boldsymbol{x}_{m}, \boldsymbol{x}^{\prime}\right) \cdot \boldsymbol{J}\left(\boldsymbol{x}^{\prime}\right) d S^{\prime}
\end{aligned}
$$

where in (4) the observation point is on the $m$ th target, and the integration is performed over the surface of target $k$.

One could solve (3) directly, although we have found it easier to consider the following iterative procedure. The efficacy of the iterative approach is discussed further below. We now define the vector propagator from target $k$ to target $m$ as

$$
\boldsymbol{P}_{m k}\left(\boldsymbol{J}_{k}\right) \equiv-\alpha_{m} \boldsymbol{E}_{k}^{s c a t}\left(\boldsymbol{x}_{m}\right)-\eta_{i}\left(1-\alpha_{m}\right) \boldsymbol{n}_{m} \times \boldsymbol{H}_{k}^{s c a t}\left(\boldsymbol{x}_{m}\right)
$$

where we have made explicit the dependence of $\boldsymbol{P}_{m k}$ on the current on target $k, \boldsymbol{J}_{k}$. We now rewrite (3) as 


$$
\boldsymbol{t}_{m} \cdot\left[\alpha_{m} \boldsymbol{E}^{i n c}\left(\boldsymbol{x}_{m}\right)+\eta_{i}\left(1-\alpha_{m}\right) \boldsymbol{n}_{m} \times \boldsymbol{H}^{i n c}\left(\boldsymbol{x}_{m}\right)-\sum_{k \neq m} \boldsymbol{P}_{m k}\left(\boldsymbol{J}_{k}\right)\right]=\boldsymbol{t}_{m} \cdot\left[\eta_{i}\left(1-\alpha_{m}\right) \boldsymbol{J}\left(\boldsymbol{x}_{m}\right)+\boldsymbol{P}_{m m}\left(\boldsymbol{J}_{m}\right)\right]
$$

The representation in (6) suggests the following iterative solution strategy. Let $\boldsymbol{J}_{k}^{q}$ represent the surface current on target $k$ on iteration $q$, where we start at $q=0$. Defining $U(q=0)=0$ and $U(q>0)=1$, we solve (6) via the iterative algorithm

$$
\boldsymbol{t}_{m} \cdot\left[\alpha_{m} \boldsymbol{E}^{i n c}\left(\boldsymbol{x}_{m}\right)+\eta_{i}\left(1-\alpha_{m}\right) \boldsymbol{n}_{m} \times \boldsymbol{H}^{i n c}\left(\boldsymbol{x}_{m}\right)-U(q) \sum_{k \neq m} \boldsymbol{P}_{m k}\left(\boldsymbol{J}_{k}^{q-1}\right)\right]=\boldsymbol{t}_{m} \cdot\left[\eta_{i}\left(1-\alpha_{m}\right) \boldsymbol{J}_{m}^{q}\left(\boldsymbol{x}_{m}\right)+\boldsymbol{P}_{m m}\left(\boldsymbol{J}_{m}^{q}\right)\right]
$$

In (7), on the initial iteration $q=0$ the $M$ targets are analyzed in isolation. On subsequent iterations $q>0$ interactions between the targets are accounted for by the summation term in (7), using the currents computed on the previous iteration. For $q>0$ and a given target $m$, the left side of (7) corresponds to the incident fields plus the scattered fields from all other targets $k \neq m$, the latter computed using the currents induced on targets $k \neq m$, as computed from iteration $q-1$. In the examples we have considered, as discussed further in Secs. III and IV, we have found that the algorithm in (7) typically converges after a small number of iterations. The uniqueness theorem guarantees that it converges to the correct solution, if all boundary conditions are satisfied, as they are by construction.

\section{Discussion}

The formulation in (7) has several attractive features. For example, the method involves analysis of scattering from isolated targets, and therefore it is directly amenable to existing codes [13-21]. The interactions between the targets are accounted for by updating the excitation fields on the left side of (7). Therefore, although the formulation has been presented for multiple perfectly conducting targets, it is readily applicable to dielectric targets as well. We also note that in the formulation in Sec. IIA it has been assumed that each target exists entirely in a single region of the half space (region $i$ ). However, the formulation in (7) is also applicable to multiple targets, with a subset of such in one half-space region, and the remaining targets in the other. For this case the propagator in (5) is modified to account for the scattered fields in one region due to induced currents 
in the other.

The construction in (7) is also ideally suited for a parallel implementation. Individual processors can be used to model scattering from particular targets, with interaction between the different targets accounted for via communication between processors (represented by the summation term in (7)). Such an approach allows consideration of a large number of scatterers, analogous to the work of Tsang et al. [3] in which multiple dielectric cylinders were considered. We have not implemented such a parallel implementation here, although we have employed a related formulation that has increased the problem sizes we have addressed in this initial study.

Note that for computation of the induced currents on target $m$ at iteration $q, \boldsymbol{J}_{m}{ }^{q}$, we require the currents on all other targets $k \neq m$ at iteration $q$-1. Assume that the number of unknowns associated with target $m$ is $N_{m}$, then the required RAM for computation of the induced currents on target $m$ is order $N_{m} \log N_{m}$ (for the MLFMA) plus order $\sum_{k \neq m} N_{k}$ (for storage of the currents on all other targets, computed on the previous iteration). The latter memory requirements are typically significantly smaller than that of the former. Therefore, within the context of the results presented in Secs. III and IV, our software is written such that, when computing the induced currents for target $m$ at iteration $q$, we store the currents induced on the other targets at iteration $q$-1, but not their associated MLFMA components. This implies that the composite target we consider on a single processor can be larger than if the entire problem were solved with a direct MLFMA solver. In particular such a direct MLFMA solution requires order $\left(\sum_{k} N_{k}\right) \log \left(\sum_{k} N_{k}\right)$ memory.

We note an additional attractive feature of the algorithm in Secs. IIA and IIB. On the initial iteration $q=0$, no interactions are accounted for between targets. We therefore know that for $q=0$ the computed target currents are highly approximate (ignoring multiple interactions), with the overall accuracy increasing gradually for increasing $q$. For iteration $q$, a matrix equation of the form $\boldsymbol{Z}_{m} \boldsymbol{i}_{m}{ }^{q}=\boldsymbol{v}_{m}{ }^{q-1}$ is solved via the MLFMA, for each target $m$, where we emphasize that the driving function $v_{m}^{q-1}$ employs the currents on the other targets computed at iteration $q-1$. The solution is obtained via an iterative solver (e.g. the conjugate-gradient method [12]). As indicated above, it is known a priori that, for small $q$, all target-target interactions are not accounted for rigorously, and 
therefore it is unnecessary to solve $\boldsymbol{Z}_{m} \boldsymbol{i}_{m}{ }^{q}=\boldsymbol{v}_{m}{ }^{q-1}$ (via the MLFMA) to the accuracy one desires of the final solution. Therefore, for small $q$ the MLFMA error requirement $\left|\boldsymbol{Z i}^{q}-\boldsymbol{v}^{q-1}\right| /\left|\boldsymbol{v}^{q-1}\right|$ for CG termination is set to be relatively large, with the required error diminishing gradually as $q$ increases. The initial relaxed MLFMA error requirement accelerates CG convergence, without sacrificing (in our experience) final algorithm accuracy. For $q=0,1,2,3,4$ we have set the (percentage) MLFMA error criteria at $10 \%, 5 \%, 1 \%$ and $0.5 \%$, respectively. For $q>5$ the error criterion is fixed at $0.1 \%$. The overall algorithm is terminated when all the currents converge, i.e. when $\left|\boldsymbol{i}^{q} \boldsymbol{i}^{q-1}\right| /\left|\boldsymbol{i}^{q-1}\right|$ is below a prescribed value, where here we have chosen $0.1 \%$.

\section{Canonical Examples}

Before presenting scattering examples of interest to remote sensing, we first consider three canonical examples. The first example considers the MLFMA analysis of scattering from two perfectly conducting cylinders residing in free space. This problem is solved in two ways: (i) with both targets treated as a single composite target (as in a traditional MLFMA analysis) and (ii) using the iterative scheme discussed in Sec. II. In addition to comparing the scattered fields computed via these two formalisms, we address the required computer memory and computation time.

Both of the aforementioned approaches employ the MLFMA, and therefore the second example is used for validation of the formulation discussed in Sec. II, with a comparison of the scattered fields made with an independent algorithm. In particular, we compare results from the iterative MLFMA with scattering data computed using a time-domain algorithm: the multi-resolution time-domain (MRTD) [26]. The MRTD formulation is similar to the widely used finite-difference time-domain (FDTD) algorithm [27]. However, while FDTD expands the fields in a pulse basis, the MRTD employs a wavelet basis [26]. The interested reader is referred to [26] for further details on the MRTD formulation.

The final example in this section considers scattering from two dielectric cylinders situated above a dielectric half space. The results from this canonical example can be used in the future by other investigators as a reference for newly developed models. 


\section{A. Perfectly conducting cylinders in free space}

We consider the two cylindrical targets depicted in Fig. 1, each target a perfect electric conductor (PEC). The incident angles are $\theta_{i}=60^{\circ}$ and $\phi_{i}=30^{\circ}$, and the bistatic scattered fields are observed for $\theta_{s}=60^{\circ}$ and $-180^{\circ} \leq \phi_{s} \leq 180^{\circ}$. A comparison is shown in Fig. 2 between scattering results computed via the direct and iterative MLFMA models, with excellent agreement demonstrated for the co-polarized and cross-polarized field components. These results are computed for operation at $600 \mathrm{MHz}$, with the unknowns on each of the two targets $N_{1}=N_{2}=6825$ (a total of 13,650 unknowns across both targets). For this relatively small problem the iterative MLFMA required 240 Mbytes of RAM, while the direct MLFMA require 660 Mbytes, demonstrating the potential of the iterative algorithm to address larger scattering problems, with a given RAM. The iterative algorithm converged after $q=6$ iterations, and the total required CPU time of the two formulations was found to be approximately the same.

Concerning convergence of the iterative algorithm discussed in Sec. II, in Fig. 3 we plot the change in the currents $\left|\boldsymbol{i}_{m}{ }^{q}{ } \boldsymbol{i}_{m}{ }^{q-1}\right| /\left|\boldsymbol{i}_{m}{ }^{q-1}\right|$ for each of the targets $(m=1$ and $m=2)$. We see that the iterative algorithm converges quickly in this example, with similar convergence having been found for all examples considered thus far.

\section{B. Two dielectric cylinders in free space}

We consider the bistatic radar cross section (RCS) for two proximate dielectric cylinders situated in free space. The scattering geometry is shown inset in Fig. 4(b). The relative permittivity of each target is $\epsilon_{r}=3$. In Fig. 4 we compare the bistatic RCS as computed via MRTD [26] and via the iterative MLFMA model, for a frequency of $700 \mathrm{MHz}$, with incident angles $\theta_{i}=45^{\circ}$ and $\phi_{i}=0^{\circ}$, with observation at the angles $\theta_{s}=90^{\circ}$ and $0^{\circ} \leq \phi_{i} \leq 180^{\circ}$. While one is unlikely to perform such a bistatic measurement in practice, we have found bistatic comparisons to be a particularly good test of model accuracy. The MLFMA results are computed in the frequency domain, as discussed in Sec. II, and the MRTD results are found from a Fourier transform of the associated time-domain scattered fields. 
The agreement reflected in Fig. 4 between the two models is generally good, although there are some differences at particular angles. Note that the discretization procedures for the MRTD and MLFMA models are entirely different, and we attribute most of the differences in the results to this fact (as applied here the MLFMA applies surface basis functions, while the MRTD applies volumetric wavelet-based basis functions). In particular, as the discretization rate of the two models was increased, the agreement between the two solutions improved accordingly. The MRTD results in Fig. 4 correspond to a sample rate of 8 to 14 basis functions per wavelength in free space, while the MLFMA results with RWG basis functions [24] sample the target surface with approximately ten samples per wavelength in free space. For the MLFMA solution target one required $N_{1}=8,256$ unknowns, and target two required $N_{2}=5,916$.

\section{Cylinders over a half space}

In our next example we consider two identical dielectric cylinders, of $96 \mathrm{~cm}$ height, $40 \mathrm{~cm}$ diameter and dielectric constant $\epsilon_{r}=3.0$. The cylinders are sitting atop a lossy dielectric half space, with their axes perpendicular to the half-space interface, with the target axes separated by $70 \mathrm{~cm}$. The soil is characterized by $\epsilon_{r}=5-j 0.2$ and $\sigma=0.01 \mathrm{~S} / \mathrm{m}$, and the operating frequency is $600 \mathrm{MHz}$. In Fig. 5 we present bistatic RCS results, for $\theta_{i}=45^{\circ}, \theta_{s}=45^{\circ}$, and $0^{\circ} \leq \phi_{s} \leq 180^{\circ}$ (the coordinate systems is as in Fig. 1). We consider azimuthal incident angles $\phi_{i}=0^{\circ}, 30^{\circ}, 60^{\circ}$ and $90^{\circ}$.

For the results presented in Fig. 5 each target employed eight basis functions per wavelength, entailing $N_{1}=N_{2}=11,364$ unknowns. These computations were performed on a $550 \mathrm{MHz}$ Pentium III computer with 1 GBytes of RAM. Note that, within the context of the MLFMA, the analysis of each target individually requires 0.93 GBytes of RAM, and therefore it was the iterative algorithm discussed in Sec. II that allowed us to characterize composite scattering from these two targets with the available RAM.

\section{Remote-Sensing Examples}

The examples considered in Sec. III are of interest for validating our model (Figs. 2 and 4) 
and for generation of example results that may be of interest to future investigators validating new algorithms (Fig. 5). However, more-complex scattering problems are of interest for remote-sensing applications. In this section we consider four such examples. These examples demonstrate the utility of the algorithm, although the results themselves may be of limited quantitative value since other investigators will likely not have access to the meshes with which these results were computed. Nevertheless, the examples presented here demonstrate the utility of the iterative MLFMA formalism in the context of analyzing realistic scattering scenarios.

\section{A. Concealed man-made target}

We consider plane-wave scattering from a model T72 tank situated above soil characterized by $\epsilon_{r}=5-j 0.01$ and $\sigma=0.01 \mathrm{~S} / \mathrm{m}$, with operation at $300 \mathrm{MHz}$. The target mesh is shown in Fig. 6, along with a tree-like target. The "tree" is composed of multiple connected dielectric cylinders of different lengths and diameters, with this meant to represent the trunk and principal branches of a tree. At the VHF frequencies of interest here the smaller branches and leaves cause minimal perturbation to the incident fields. For simplicity the "tree" is model with a homogeneous relative permittivity $\epsilon_{r}=4$ and conductivity $\sigma=0.01 \mathrm{~S} / \mathrm{m}$. These parameters are not necessarily representative any particular tree, this simply serving as an example of the code potential. The principal dimensions of the "tank" are $8 \mathrm{~m}$ in length, $4 \mathrm{~m}$ in width, and $3 \mathrm{~m}$ in height. The diameter of the "tree" trunk is $50 \mathrm{~cm}$ and the peak height is $7.2 \mathrm{~m}$. We consider scattering from the "tank" with and without the "tree" present. When the "tree" is present (see Fig. 6) its axis is situated $3 \mathrm{~m}$ from the "tank" center. The plane-wave incidence angles are $\theta_{i}=45^{\circ}$ and $\phi_{i}=90^{\circ}$. The "tank" entails $N_{1}=12,204$ unknowns, and the "tree" $N_{2}=16,134$. The computations were performed on a $500 \mathrm{MHz}$ Pentium III computer, with 2 GBytes of RAM, the computations requiring 1.3 GBytes of RAM and approximately 100 hours of CPU.

In Fig. 7 we present the normalized induced electric current $\boldsymbol{J} /\left|\boldsymbol{n} \times \boldsymbol{H}^{i n c}\right|$ on the "tank", for vertical (V) and horizontal $(\mathrm{H})$ polarization excitation, when the "tree" is and is not present. For both polarizations we see the effects of "tree" blockage, with this appearing more prominently for $\mathrm{H}$ polarization. A comparison of the bistatic RCS, with and without the "tree", is shown in Fig. 8 for $\theta_{s}=45^{\circ}$ and $-180^{\circ} \leq \phi_{s} \leq 180^{\circ}$. 
To save space we do not plot the error $\left|\boldsymbol{i}_{k}^{q}{ }^{q} \dot{\boldsymbol{i}}_{k}^{q-1}\right| /\left|\boldsymbol{i}_{k}^{q}\right|$ for this example, however the convergence is similar to that reflected in Fig. 3.

\section{B. Two "trees"}

In our next example we consider scattering from two tree-like targets. In particular, we consider the same "tree" as addressed in Figs. 7-8, now in the context of two trees. Let $\boldsymbol{M}_{1}$ represent the mesh for the "tree" considered in Figs. 7-8. In the context of two "trees" we consider the mesh $\boldsymbol{M}_{1}$ and a shifted and rotated version of $\boldsymbol{M}_{1}$ (see Fig. 9). The formulation developed here is applicable to general such meshes, these chosen as a simple example. We consider the same soil characteristics as in Figs. 7 and 8, with operation at $300 \mathrm{MHz}$, and plane-wave incidence at angles (see coordinate system in Fig. 9) $\theta_{i}=45^{\circ}$ and $\phi_{i}=90^{\circ}$.

For this incidence direction we see that one tree is strongly shielded by a tree in front of it, and it is of interest to examine the effects of such blockage. In Fig. 10 we plot the induced electric currents on the dielectric "tree" scatterer, when the tree is in isolation and when it is shielded by a tree in front of it. Results are shown for both vertical (V) and horizontal $(\mathrm{H})$ polarization, with the effects of tree shielding more evident for $\mathrm{H}$ polarization. This is consistent with the results in Sec. IVA, in which the tank currents were perturbed for $\mathrm{H}$ polarization more than they were for $\mathrm{V}$ polarization, when partially shielded by the same "tree".

In Fig. 11 we plot the bistatic RCS for the same incident conditions discussed above, for scattering angles $\theta_{s}=45^{\circ}$ and $-180^{\circ} \leq \phi_{s} \leq 180^{\circ}$. Results are shown in Fig. 11 for scattering from the single tree (when there is no blockage) and when the two trees are present. In Fig. 11 are seen the expected strong frequency-dependent interference effects induced by scattering from multiple targets (this is seen to a far less extent in the single-tree results, although in that case multiple scattering is possible among the branching structure).

\section{Two buried unexploded ordnance}


In Fig. 12 we depict the mesh used to simulate scattering from two unexploded ordnance (UXO) buried in soil, using the same soil properties as used in the previous examples. In Fig. 12 the air-soil interface is situated at $z=0$. The incident angles (see coordinate system in Fig. 12) are $\theta_{i}=60^{\circ}$ and $\phi_{i}=120^{\circ}$, and the bistatic scattering angles are $\theta_{s}=60^{\circ}$ and $-180^{\circ} \leq \phi_{s} \leq 180^{\circ}$. For this example, we consider two sets of results: (i) the RCS computed rigorously, via the iterative formulation discussed in Sec. II; and (ii) the RCS computed by treating each UXO in isolation, and simply adding their signatures (ignoring interaction). The latter approach is expected to yield reasonable results, because the interaction effects are diminished by propagation through the lossy ground. The RCS results in Fig. 13, for operation at $600 \mathrm{MHz}$, indicate that the simple linear-combination (no interactions) model can predict the general RCS variation with angle, although the detailed RCS can be off by several dB. In this example $N_{1}=N_{2}=8,295$.

\section{Target under a rough soil interface}

All of the previous examples have considered targets in free space or in one region of a halfspace environment. The formulation in Sec. II can also be applied to the problem of targets in different regions of the half space environment. In particular, we consider the problem of a buried target under a rough air-soil interface. The PEC subsurface target is modeled as in the previous examples. The rough surface is modeled as a closed dielectric target sitting atop an infinite lossy dielectric half space. The dielectric properties of this target are identical to those of the infinite soil subsurface, and the dielectric target is placed in direct contact with the half space. A smooth taper is used to transition the surface roughness to the infinite half space, and plane-wave excitation is applied. We needn't apply Gaussian-beam excitation [28], because our rough surface is embedded in an infinite half space, mitigating the edge effects associated with previous work in which the rough interface is not closed [28]. In [29] we have discussed the MLFMA analysis of a single dielectric target placed in direct contact with a half-space interface.

In Fig. 14 we plot the mesh for a perfectly conducting target situated under a rough air-soil interface. With regard to the rough-interface mesh, this figure only depicts the top of the closed surface, but it is closed on the bottom by a flat surface, the latter in direct contact with the interface 
of the infinite half space. The soil and dielectric target (rough interface) are characterized by complex permittivity $\epsilon_{r}=5-j 0.2$ and conductivity $\sigma=0.01 \mathrm{~S} / \mathrm{m}$. Results are presented for an operating frequency of $300 \mathrm{MHz}$. The surface-roughness height is characterized by (in units of meters)

$$
h(x, y)=0.4\left[1-\cos \left(8 \pi x / x_{\max }\right)\right]\left[1-\cos \left(8 \pi y / y_{\max }\right)\right]
$$

where $x_{\max }=y_{\max }=1.8 \mathrm{~m}$. The cylindrical target has a length of $1.5 \mathrm{~m}$ and a diameter of $60 \mathrm{~cm}$, with the target situated at a depth of $40 \mathrm{~cm}$, from the target axis to the half-space interface. The dielectric target is composed of $N_{1}=14,760$ unknowns, and the subsuraface target has $N_{2}=1,515$ unknowns. The rough surface of a realistic problem must be treated as a random process, with this example simply shown to demonstrate the potential of the formulation.

In Fig. 15 we plot the co-polarized and cross-polarized bistatic RCS, for incidence angles $\theta_{i}=45^{\circ}$ and $\phi_{i}=0^{\circ}$, and the scattered fields are observed at $0^{\circ} \leq \theta_{s} \leq 90^{\circ}$ and $\phi_{i}=0^{\circ}$. The scattered fields of the target are shown with and without the rough interface. For the parameters chosen the rough surface introduces a significant perturbation of the total scattered fields.

\section{Conclusions}

An iterative procedure has been developed for extending the MLFMA to the case of multiple targets, while retaining the basic MLFMA construct. In particular, each of multiple targets is analyzed in isolation via an iterative procedure, with excitation equal to the incident fields plus the scattered fields from all other targets. Concerning the latter, these scattered fields are computed using the target-dependent induced currents computed on the previous iteration. In the implementation presented here, all computations have been performed on a single computer processor. However, to reduce memory requirements, we only utilize the MLFMA components required for the target

analyzed on a given iteration. In this manner the memory requirements are dictated primarily by the electrical size of the largest target considered, rather than by the cumulative electrical size of all targets.

The accuracy of the model has been assessed through comparison with a direct MLFMA 
analysis of multiple targets and by comparison to scattering data computed via an unrelated model (MRTD [26]). In all cases the accuracy of the model was found to be good, and the computation time of the iterative analysis was comparable to that of the direct MLFMA solver (although, as indicated above, the iterative solver required less computer memory).

Having validated the model, it was applied to demonstrate its applicability to several remotesensing applications. In particular, it was used to analyze scattering from a model tree and tank, two trees, two buried unexploded ordnance, and for a target buried under a rough air-soil interface. In the future the model will be extended to a parallel implementation, in which each of the multiple targets are analyzed simultaneously on different computer processors, with the scattered fields from each communicated to all other computer processors, to update the excitation fields on each algorithmic iteration. With such a formulation, in which one can exploit the potential of tens of parallel computer processors, one can envision consideration of complex problems involving, for example, many trees, an electrically large air-soil interface, and many surface or buried unexploded ordnance or land mines.

\section{References}

[1] T. Tamir, "On radio-wave propagation in forest environments, IEEE Trans. Antennas Propagat., vol. AP-15, pp. 806-817, 1967.

[2] D. Dence and T. Tamir, "Radio loss of lateral waves in forest environments," Radio Sci., vol. 4, pp. 307-318, 1969.

[3] L. Tsang, K.H. Ding, G. Zhang, C. Hsu and J.A. Kong, "Backscattering enhancement and clustering effects of randomly distributed dielectric cylinders overlying a dielectric half space based on Monte-Carlo simulations," IEEE Trans. Antennas Propagat., vol. 43, pp. 488-499, May 1995.

[4] L. Tsang, K.H. Ding, G. Zhang, C. Hsu and J.A. Kong, "Backscattering enhancement and clustering effects of randomly distributed dielectric cylinders overlying a dielectric half space based on Monte-Carlo simulations," IEEE Trans. Antennas Propagat., vol. 43, pp. 488-499, May 1995. [5] L. Tsang, C.H. Chan, J.A. Kong and J. Joseph, "Polarimetric signatures of a canopy of dielectric cylinders based on first and second order vector radiative transfer theory," J. Electrom. Waves Appl., 
vol. 6, pp. 19-51, 1992.

[6] K. Sarabandi, P.F. Polatin and F.T. Ulaby, "Monte Carlo simulation of scattering from a layer of vertical cylinders," IEEE Trans. Antennas Propagat., vol. 41, pp. 465-475, 1993.

[7] G. Zhang, L. Tsang and Z. Chen, "Collective scattering effects of trees generated by stochastic Lindenmayer systems," Microwave Optical Tech. Letts., vol. 11, pp. 107-111, Feb. 5, 1996.

[8] S.H. Yueh, J.A. Kong, J.K. Jao, R.T. Shin and T. LeToan, "Branching model for vegetation," IEEE Trans. Geosci. Remote Sens., vol. 30, pp. 390-402, 1992.

[9] Z. Chen, "Microwave remote sensing of vegetation: stochastic Lindenmayer systems, collective scattering effects, and neural network inversions," Ph.D. dissertation, Dept. Of Electrical Engineering, University of Washington, 1994.

[10] J. He, T. Yu, N. Geng and L. Carin, "Method-of-moments analysis of electromagnetic scattering from a general three-dimensional dielectric target embedded in a multi-layered medium," to appear in Radio Science, Feb. 2000.

[11] J. He, N. Geng, L. Nguyen and L. Carin, "Rigorous modeling of Ultra-Wideband VHF scattering from tree trunks over flat and sloped terrain," accepted for publication in IEEE Trans. Geosci. Remote Sens.

[12] G.H. Golub and C.F. Van Loan, Matrix Computations, Johns Hopkins Univ Pr, Dec. 1996.

[13] N. Geng, A. Sullivan and L. Carin, “ Fast multipole method for scattering from an arbitrary PEC target above or buried in a lossy half space," IEEE Trans. Antennas Propagat., vol. 49, pp. 740-748, May 2001.

[14] J.Q. He, A. Sullivan and L. Carin, "Multilevel fast multipole algorithm for three-dimensional dielectric targets in the vicinity of a lossy half space," Microwave and Opt. Tech. Letts., vol. 29 (2): pp. 100-104, April 202001.

[15] N. Geng, A. Sullivan and L. Carin, "Multilevel fast-multipole algorithm for scattering from conducting targets above or embedded in a lossy half space," IEEE Trans. Geosci. Remote Sens., vol. 38, pp. 1561-1573, July 2000.

[16] J.Q. He, A. Sullivan and L. Carin, "Multilevel fast-multipole algorithm for scattering from general dielectric targets above or embedded in a lossy half space," accepted for publication in Radio Sci.

[17] X.Q. Sheng, J.M. Jin, J. Song, C.C. Lu, W.C. Chew, "Solution of combinded field integral 
equation using multilevel fast multiple algorithm for scattering by homogeneous bodies," IEEE Trans. Ant. and Prop., vol. 46, pp. 1718-1726, Nov. 1998.

[18] J.M. Song, C.C. Lu, W.C. Chew, "Multilevel fast multiple algorithm for electromagnetic scattering by large complex objects," IEEE Trans. Ant. and Prop., vol. 45, pp. 1488-1493, Oct. 1997 [19] J.M. Song, C.C. Lu, W.C. Chew and S.W. Lee, "Fast Illinois solver code (FISC)," IEEE Ant. and Prop. Magazine, vol.40, pp.27-33, June 1998.

[20] J.M. Song, W.C. Chew, "Fast Multiple method solution using parametric geometry," J. Electromagnetic Waves Appl., vol.9, pp.71-83, Jan.-Feb. 1995.

[21] J.M. Song, W.C. Chew, "Multilevel fast multiple algorithm for solving combined field integral equations of electromagnetic scattering", Micr. Opt. Techn. Lett., vol. 10, pp. 14-19, Sept. 1995. Sullivan A, Carin L

[22] A. Sullivan and L. Carin, "Scattering from complex bodies using a combined direct and iterative technique,"IEEE Trans. Ant. and Prop. Vol. 47, pp. 33-39, Jan. 1999.

[23] K.A. Michalski and D.Zheng, "Electromagnetic scattering and radiation by surfaces of arbitrary shape in layered media, Part I and II," IEEE Trans. Ant. and Prop., vol.38, pp. 335-352, March 1990. [24] S.M. Rao, D.R. Wilton, A.W. Glisson, "Electromagnetic scattering from surfaces of arbitrary shape,” IEEE Trans. Ant. and Prop., vol. 30, pp. 409-418, May 1982.

[25] R. Coifman, V. Rokhlin, S.Wandzura, "The fast multiple method for the wave equation: a pedestrian prescription," IEEE Ant. and Prop. Magazine, vol. 35, pp.7-12, June 1993.

[26] X. Zhu and L. Carin, "Multi-resolution time-domain analysis of plane-wave scattering from general three-dimensional surface and subsurface dielectric targets," accepted for publication in IEEE Trans. Antennas Propagat

[27] A. Taflove, Advances in Computational Electrodynamics : The Finite-Difference Time-Domain Method, Artech House, 1998.

[28] J.T. Johnson and R.J. Burkholder, "Coupled canonical grid/discrete dipole approach for computing scattering from objects above or below a rough interface," IEEE Trans. Geosci. Remote Sens., vol. 39, pp. 1214-1220, June 2001.

[29] Z. Liu, J. He, Y. Xie, A. Sullivan and L. Carin, "Multi-level fast multipole algorithm for general targets on a half-space interface," submitted to IEEE Trans. Ant. and Prop. 


\section{Figure Captions}

Figure 1. Two cylindrical perfect electric conductor (PEC) scatterers in free space.

Figure 2. Bistatic co-polarized radar cross section (RCS) for the targets in Fig. 1. The incident angles are $\theta_{i}=60^{\circ}$ and $\phi_{i}=30^{\circ}$, and the bistatic scattered fields are observed for $\theta=60$ ånd $180^{\circ} \leq \phi_{s} \leq 180^{\circ}$ (see Fig. 1). The curves correspond to the results of the iterative MLFMA formulation in Sec. II, and the points correspond to a direct MLFMA formulation in which the two targets in Fig. 1 are treated as a single composite target. Results are shown for plane-wave excitation at $600 \mathrm{MHz}$.

Figure 3. Relative error (or change) in the target-dependent induced currents, as a function of iteration number $q$. Explicitly, $\left|\boldsymbol{i}_{m}{ }^{q} \boldsymbol{i}_{m}{ }^{q-1}\right| /\left|\boldsymbol{i}_{m}{ }^{q-1}\right|$ is plotted as a function of iteration $q$, where $\boldsymbol{i}_{m}{ }^{q}$ represents the basis-function coefficients on target $m$ for iteration $q$. Results are shown for the example considered in Figs. 1 and 2.

Figure 4. Bistatic radar cross section (RCS) for the lossless dielectric targets shown inset in (b), with each target situated in free space and characterized by $\epsilon_{r}=3$. The points are computed via MRTD [26] and the curves via the iterative MLFMA model, for a frequency of $700 \mathrm{MHz}$, with incident angles $\theta_{i}=45^{\circ}$ and $\phi_{i}=0^{\circ}$, and observation at $\theta_{s}=90^{\circ}$ and $0^{\circ} \leq \phi_{i} \leq 180^{\circ}$. (a) co-polarized fields, (b) crosspolarized fields

Figure 5. Bistatic radar cross section (RCS) for two lossless dielectric cylinders of $40 \mathrm{~cm}$ diameter, $96 \mathrm{~cm}$ height, and relative permittivity $\epsilon_{r}=3$. The targets are situated in the top region of a half space, the top region characterized by vacuum and the lower region by $\epsilon_{r}=5-j 0.2$ and $\sigma=0.01 \mathrm{~S} / \mathrm{m}$. The bottom of each cylinder is $7 \mathrm{~cm}$ from the half-space interface, and the axes of the two targets are separated by $70 \mathrm{~cm}$. The operating frequency is $600 \mathrm{MHz}$. Results are shown for incidence angles $\theta_{i}=45^{\circ}$ and $\phi_{i}=0^{\circ}, 30^{\circ}, 60^{\circ}$ and $90^{\circ}$; and for scattering angles $\theta_{s}=45^{\circ}$, and $0^{\circ} \leq \phi_{s} \leq 180^{\circ}$ (the coordinate systems is as in Fig. 1). (a) HH polarization, (b) VV polarization

Figure 6. Mesh used to model a T72 tank situated beside a tree-like target. The soil is characterized by $\epsilon_{r}=5-j 0.2$ and $\sigma=0.01 \mathrm{~S} / \mathrm{m}$, and the "tree" by $\epsilon_{r}=4$ and conductivity $\sigma=0.01 \mathrm{~S} / \mathrm{m}$.

Figure 7. Currents induced on the tank in Fig. 6, with and without the "tree" present, for plane-wave incidence at $\theta_{i}=45^{\circ}$ and $\phi_{i}=90^{\circ}$ (see the coordinate system in Fig. 6) at a $300 \mathrm{MHz}$ operating frequency. (a) vertical polarization excitation, no tree present; (b) vertical polarization excitation, tree present; (c) horizontal polarization excitation, no tree present; (d) horizontal polarization 
excitation, tree present

Figure 8. Bistatic radar cross section (RCS) for the problem considered in Fig. 7. The scattered fields are observed at $\theta_{s}=45^{\circ}$, for $-180 \leq \phi_{s} \leq 180^{\circ}$. (a) VV polarization, (b) HH polarization

Figure 9. Mesh used to investigate scattering from multiple tree-like targets. The soil is characterized by $\epsilon_{r}=5-j 0.2$ and $\sigma=0.01 \mathrm{~S} / \mathrm{m}$, and the "trees" by $\epsilon_{r}=4$ and conductivity $\sigma=0.01 \mathrm{~S} / \mathrm{m}$.

Figure 10. Electric currents induced on the targets in Fig. 9, for the case of both trees present (as in Fig. 9) and when the "tree" situated approximately at $y=2 \mathrm{~m}$ is absent. Plane-wave incidence is considered at $\theta_{i}=45^{\circ}$ and $\phi_{i}=90^{\circ}$ (see the coordinate system in Fig. 9), for an operating frequency of $300 \mathrm{MHz}$. (a) vertical polarization excitation, (b) horizontal polarization excitation

Figure 11. Bistatic radar cross section (RCS) for the problem considered in Fig. 10. The scattered fields are observed at $\theta_{s}=45^{\circ}$, for $-180 \leq \phi_{s} \leq 180^{\circ}$. (a) VV polarization, (b) $\mathrm{HH}$ polarization

Figure 12. Mesh used to model scattering from two unexploded ordnance (UXO) buried in soil. The soil interface is at $z=0$, and the soil properties are characterized by $\epsilon_{r}=5-j 0.2$ and $\sigma=0.01 \mathrm{~S} / \mathrm{m}$.

Figure 13. Bistatic RCS for the problem depicted in Fig. 12, for plane-wave incidence at $\theta_{i}=45^{\circ}$ and $\phi_{i}=0^{\circ}$, as observed at $\theta_{s}=45^{\circ}$ and for $0^{\circ} \leq \phi_{s} \leq 180^{\circ}$. Results are shown when all interactions are accounted for via the algorithm in Sec. II, and when the targets are modeled in isolation and simply added. An operating frequency of $600 \mathrm{MHz}$ is considered.

Figure 14. Mesh used to model a rough soil interface, below which is a perfectly conducting buried target. The two targets are embedded with in infinite half space, characterized by $\epsilon_{r}=5-j 0.2$ and $\sigma=0.01 \mathrm{~S} / \mathrm{m}$.

Figure 15. Bistatic radar cross section (RCS) for the problem depicted in Fig. 14, for incidence angles $\theta_{i}=45^{\circ}$ and $\phi_{i}=0^{\circ}$, and observation at $0^{\circ} \leq \theta_{s} \leq 90^{\circ}$ and $\phi_{i}=0^{\circ}$. The scattered fields of the target are shown with and without the rough interface. Results are shown for plane-wave excitation at 300 MHz. (a) co-polarized fields, (b) cross-polarized fields 


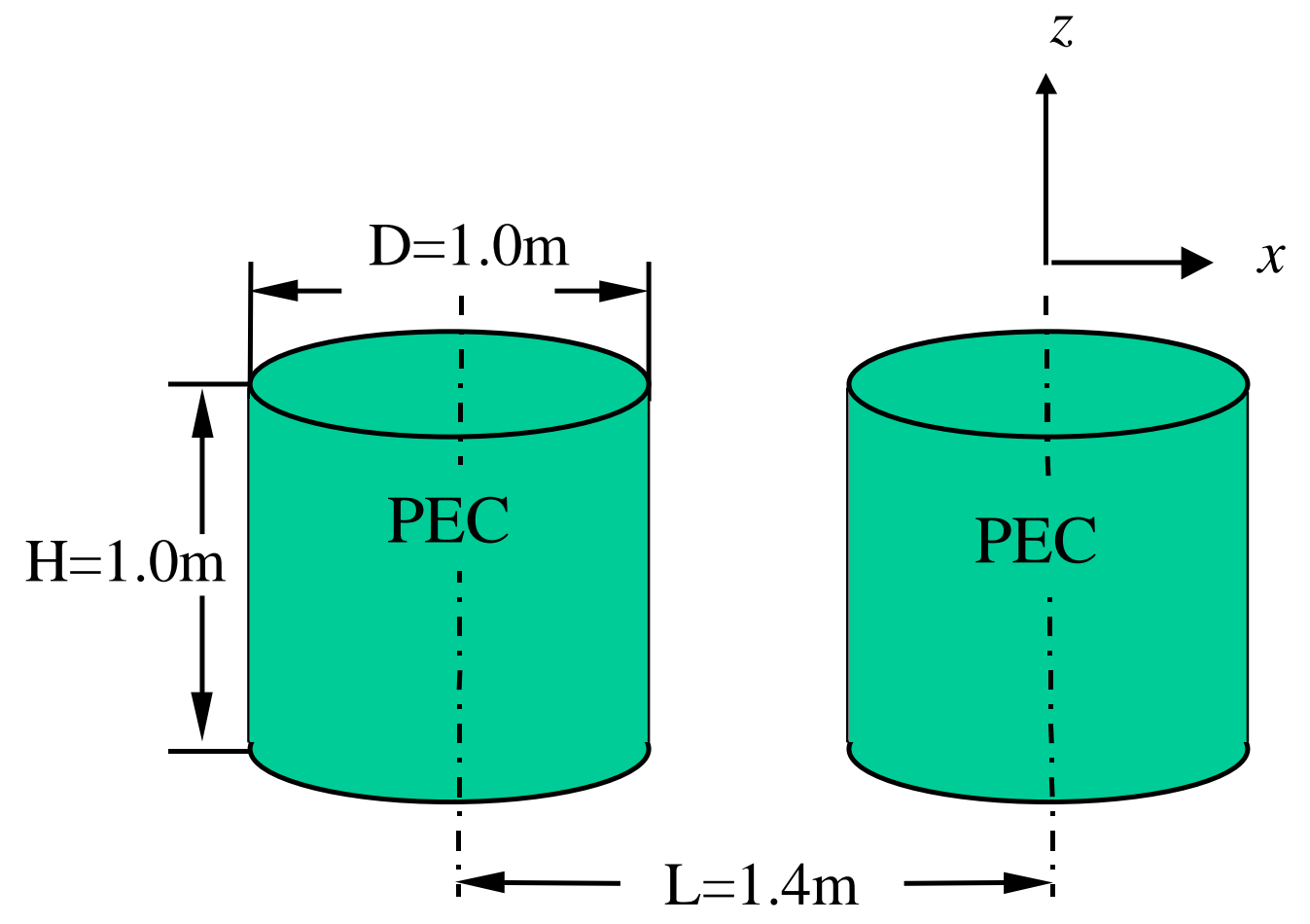

Figure 1 


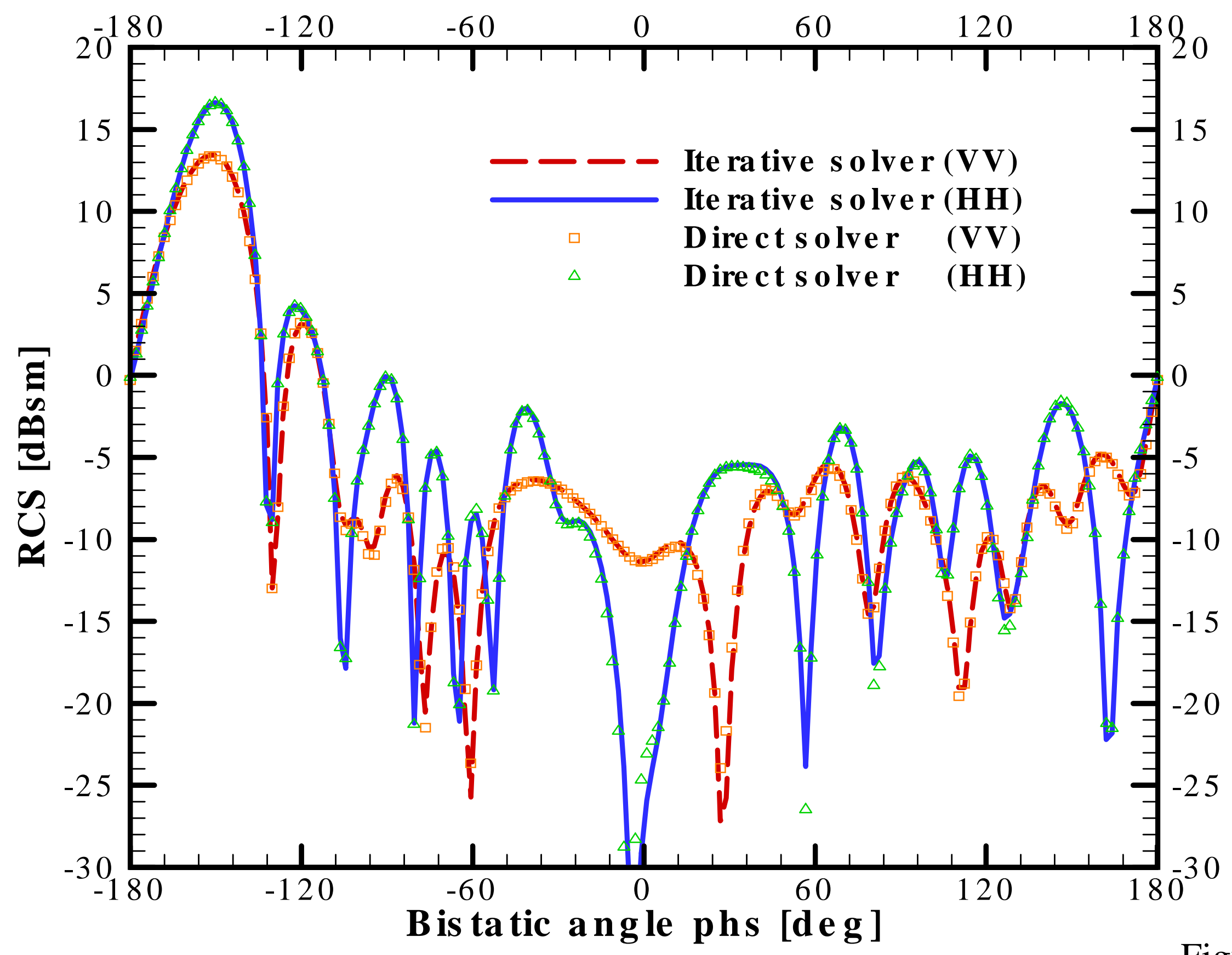

Figure 2 


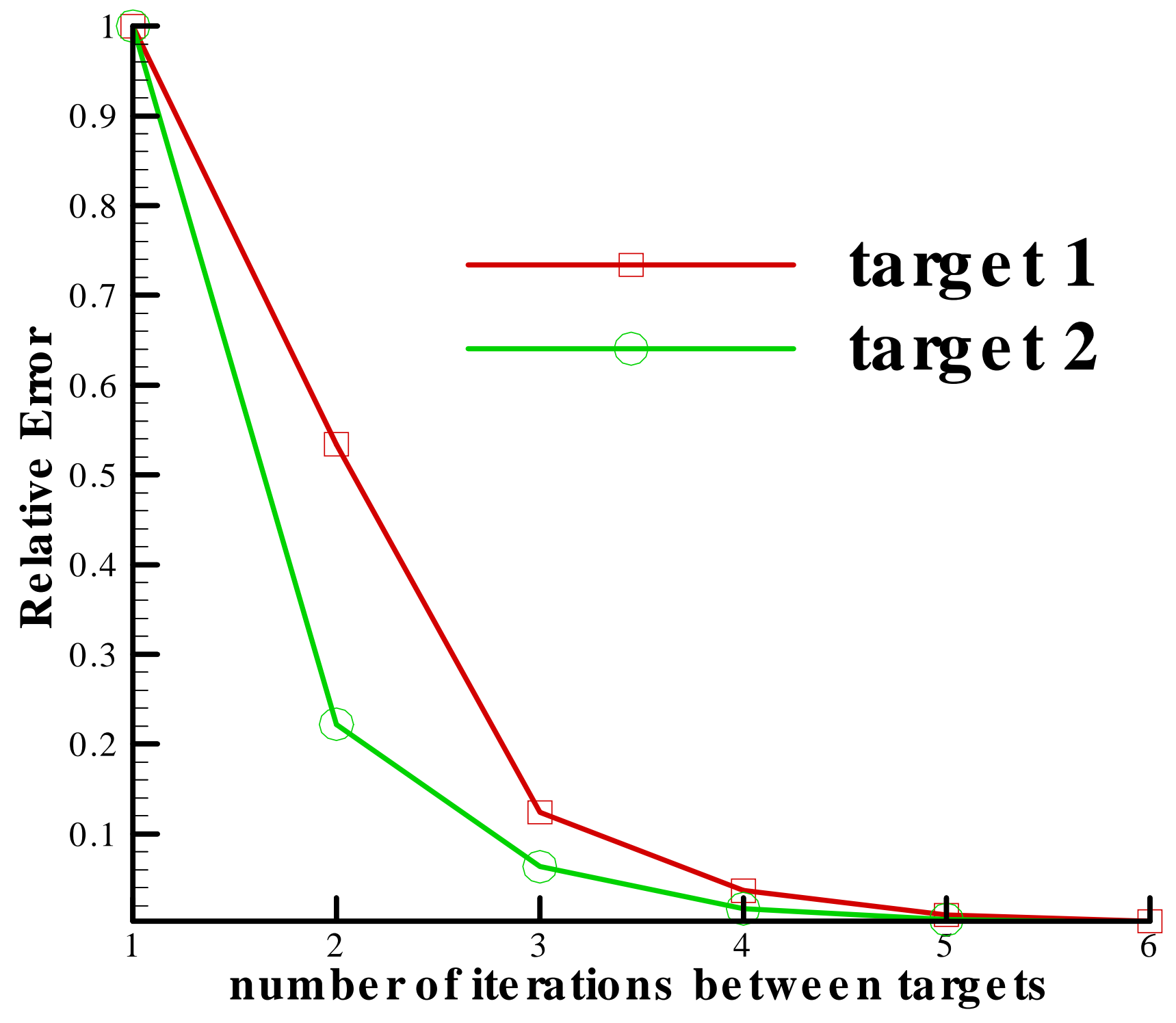

Figure 3 


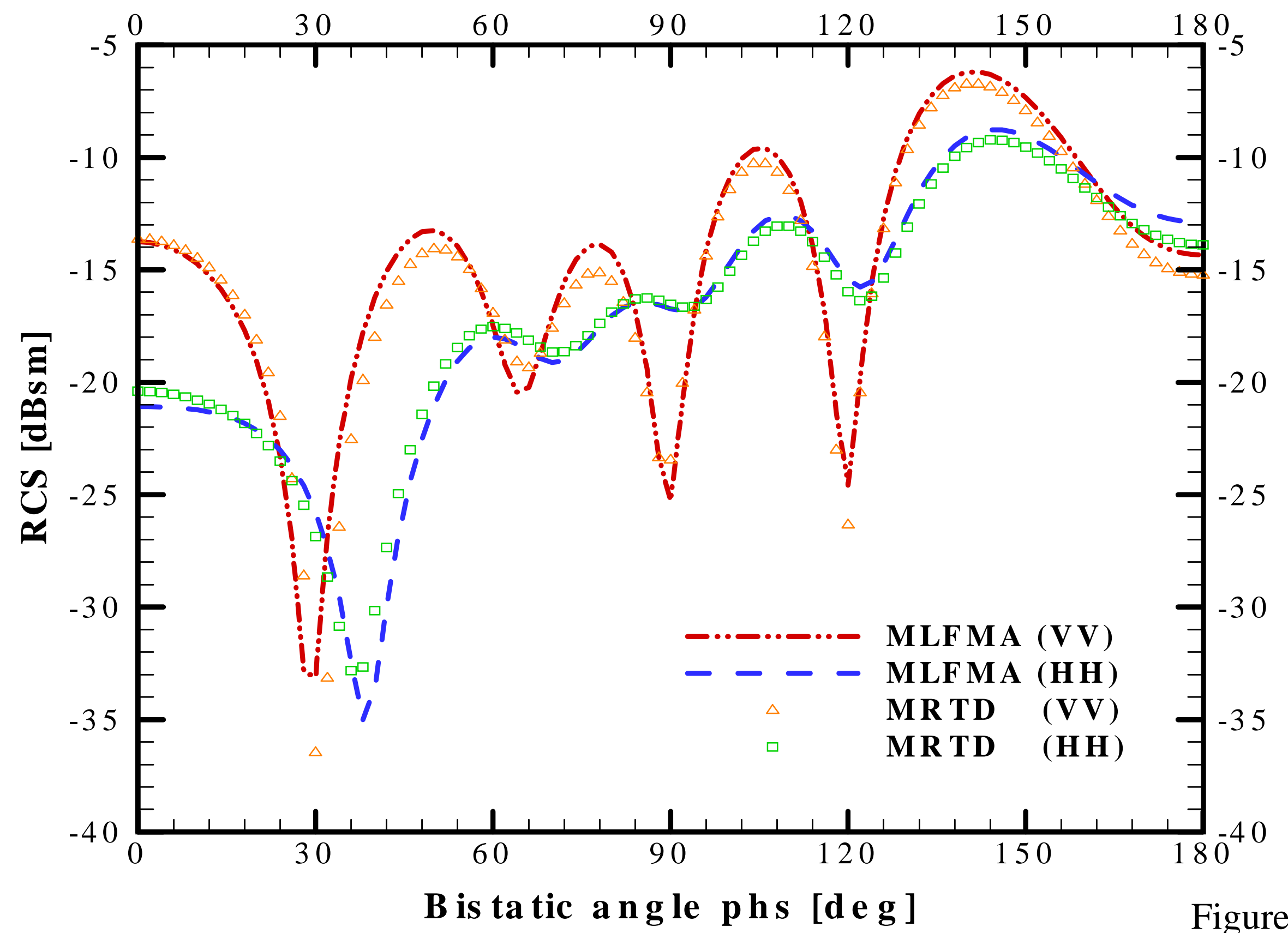

Figure 4(a) 


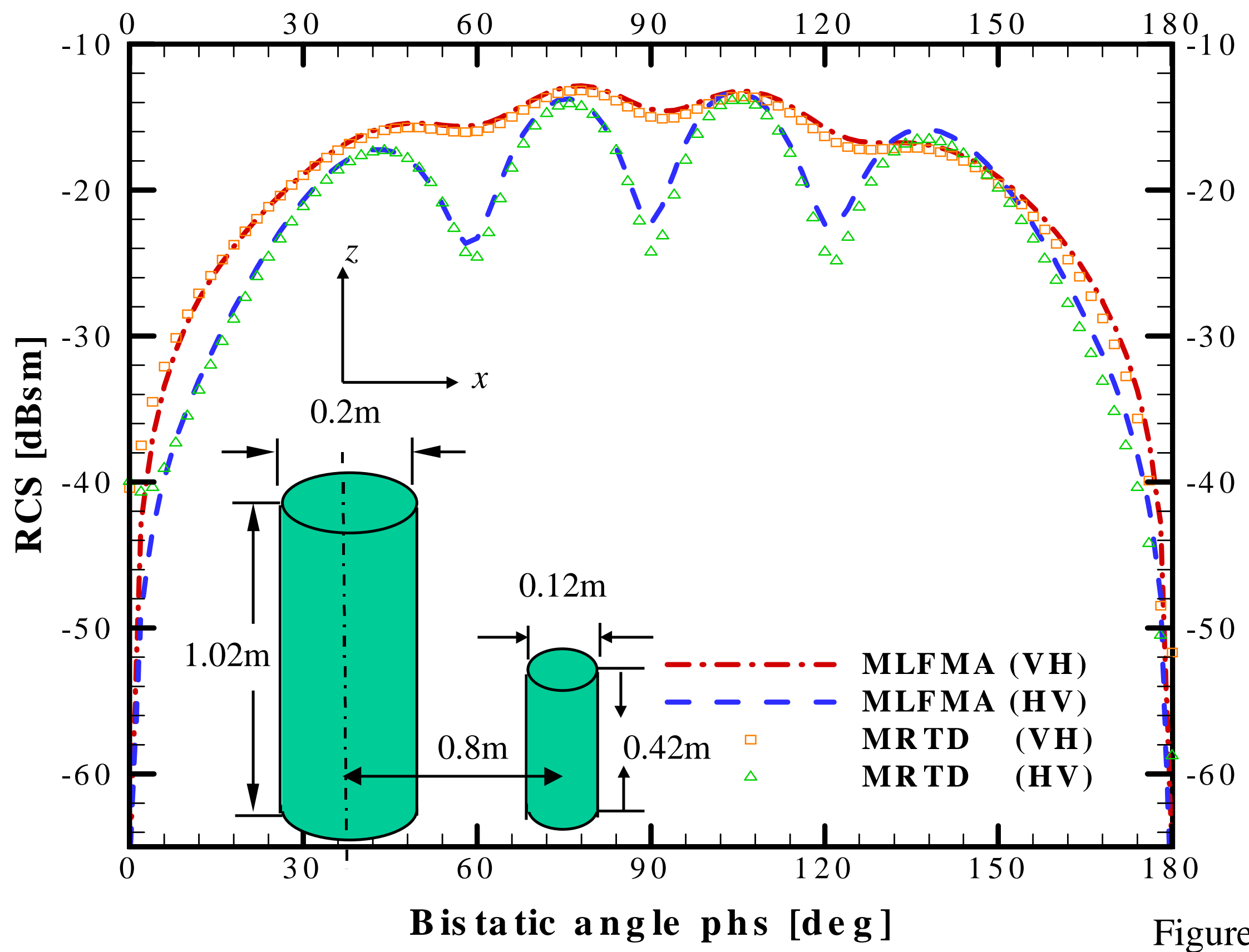

Figure 4(b) 


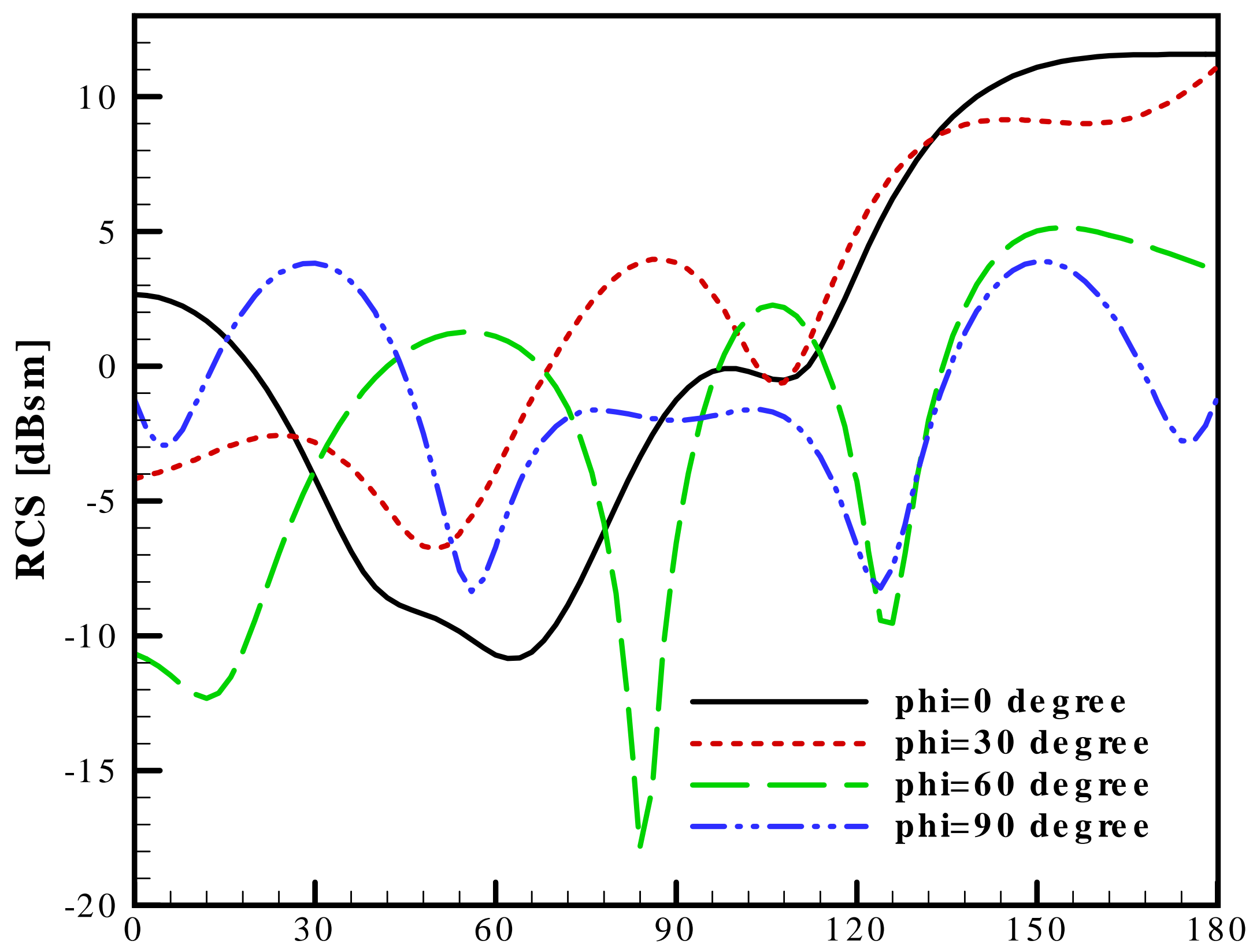




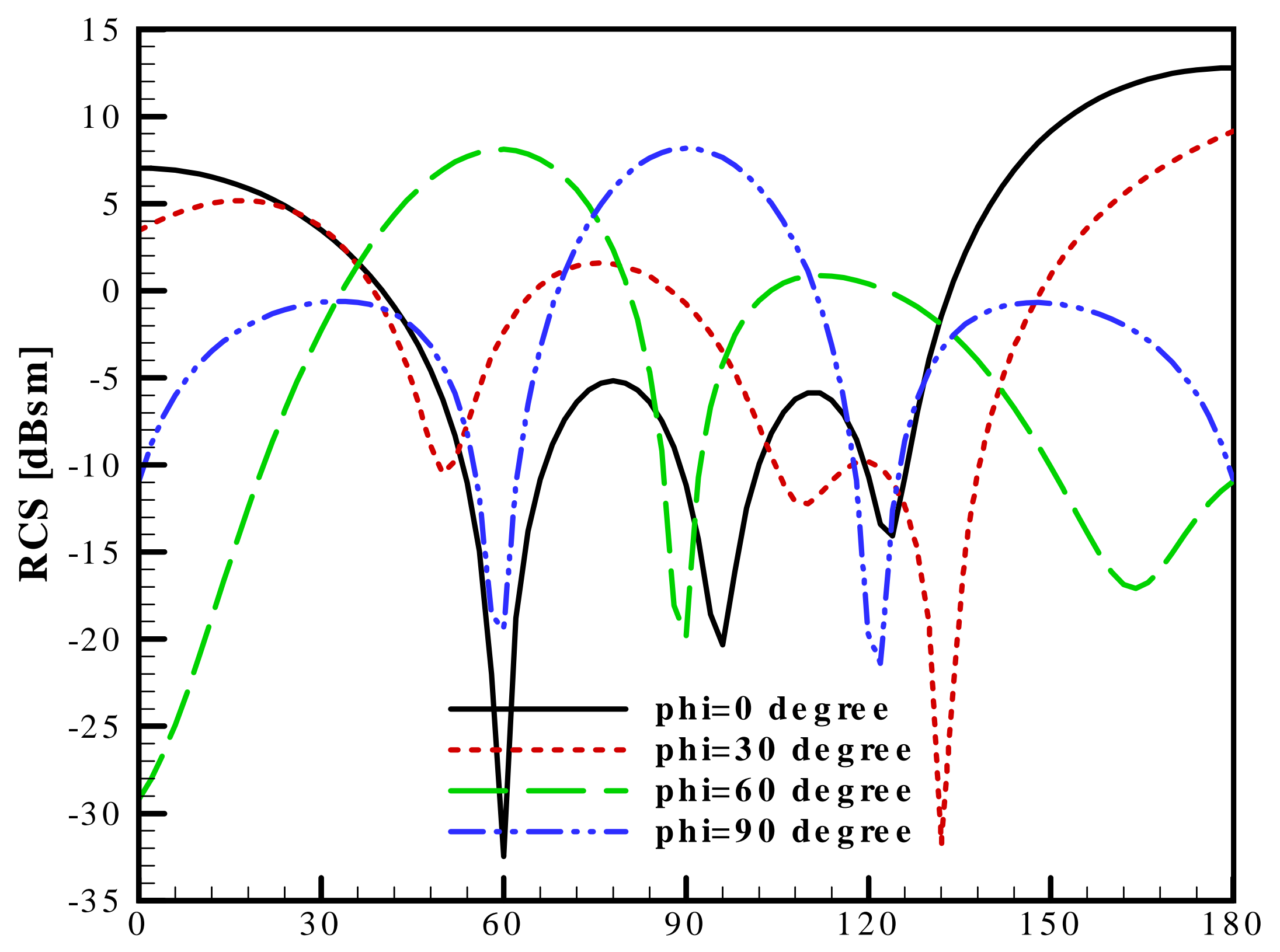




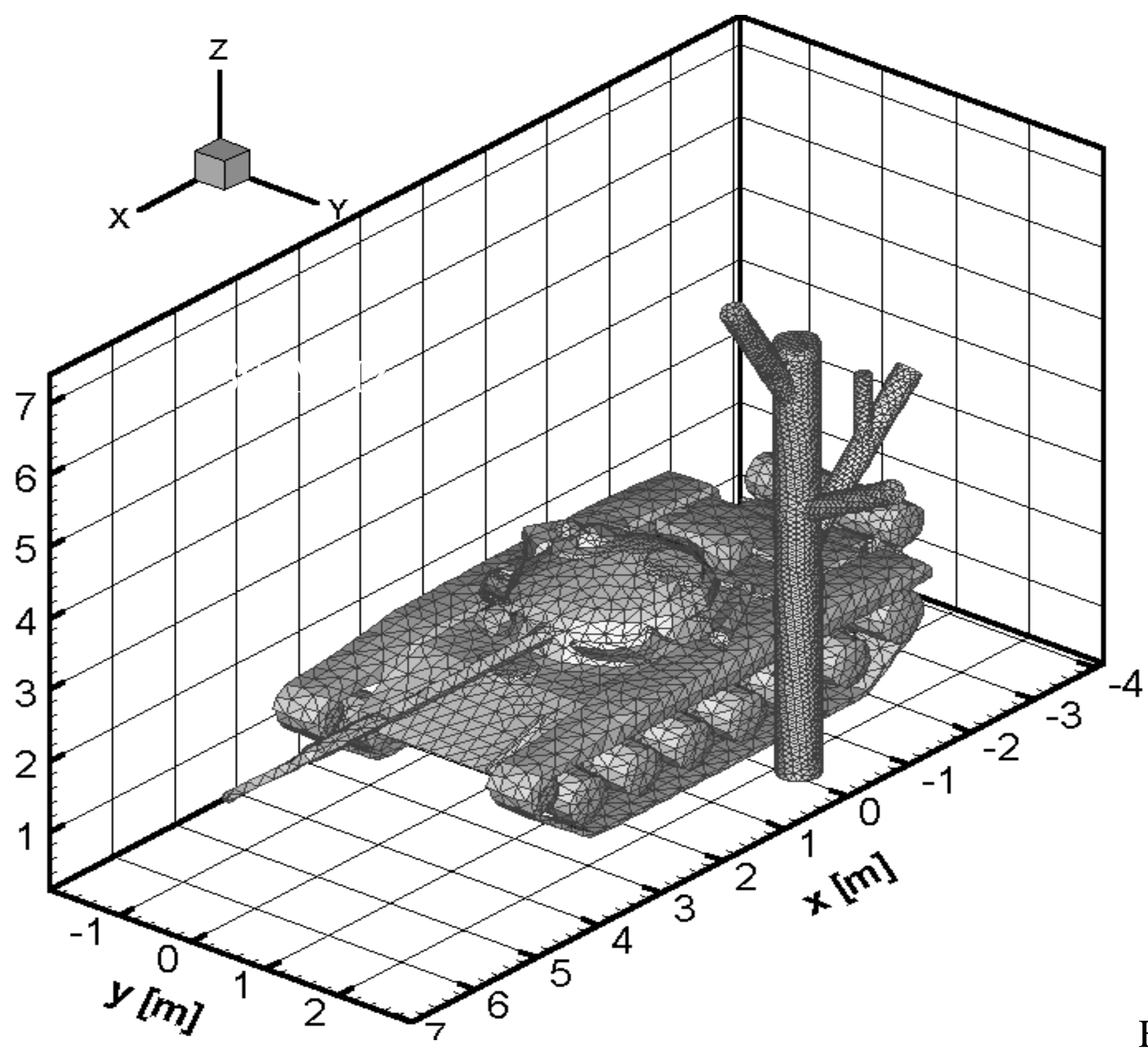

Figure 6 


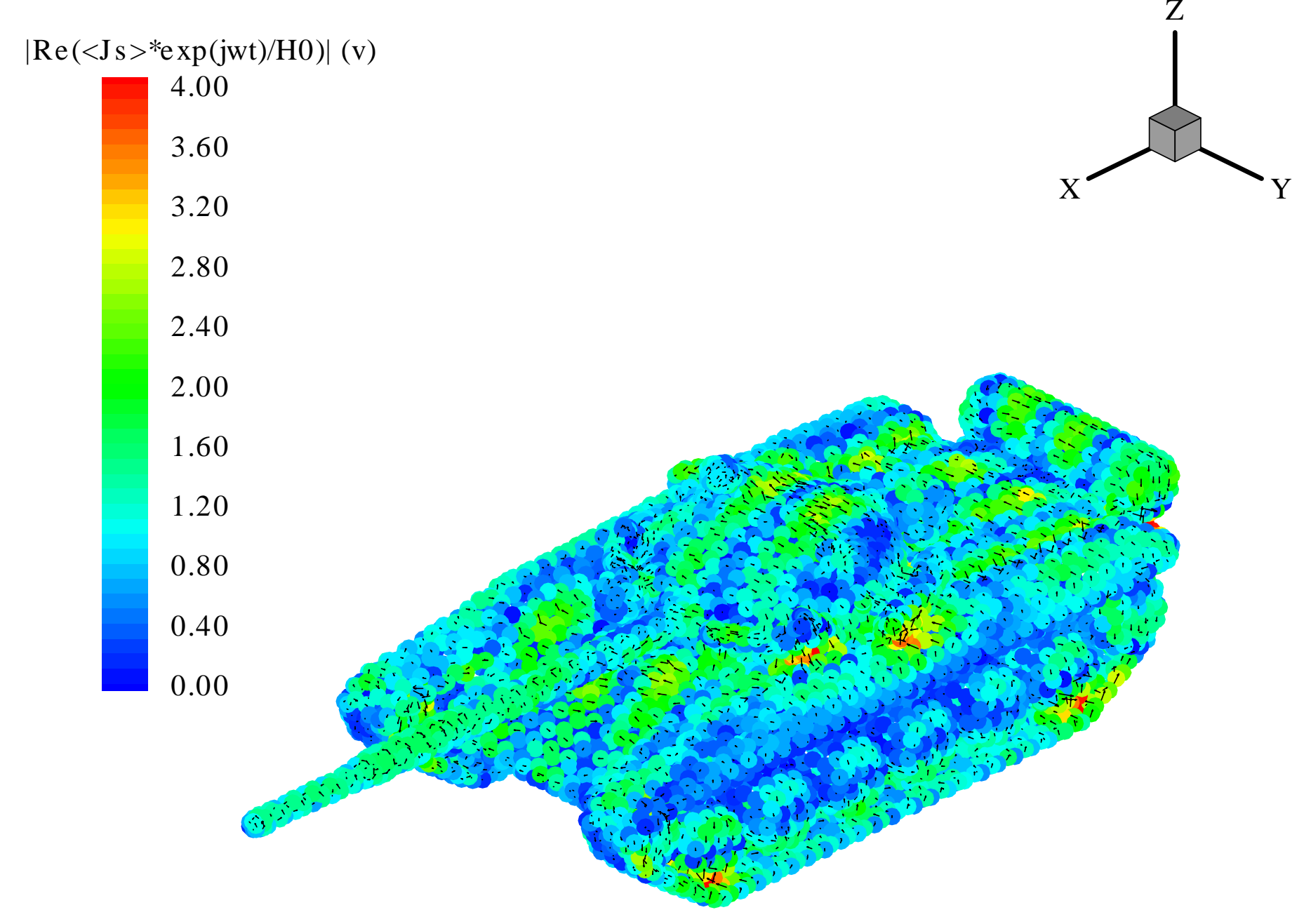

Figure 7(a) 


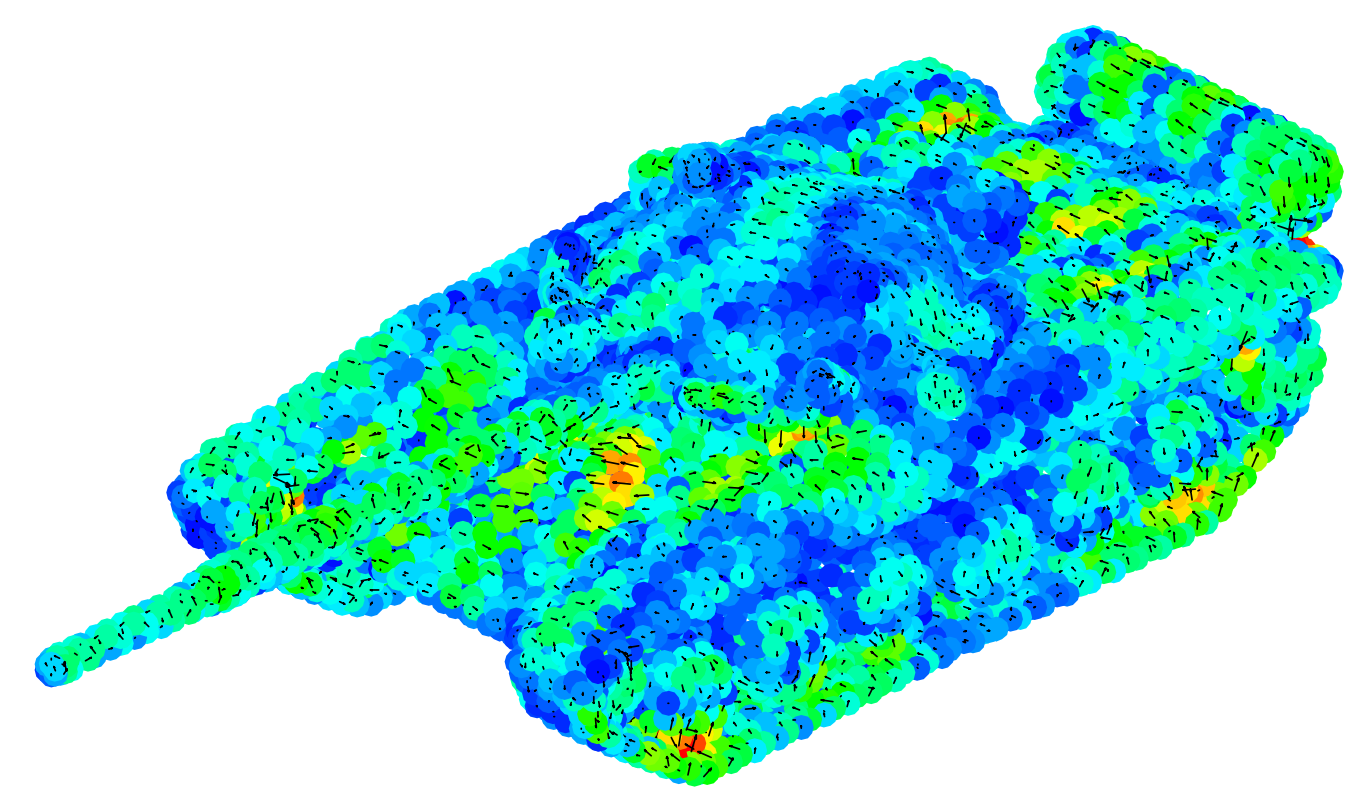

Figure 7(b) 


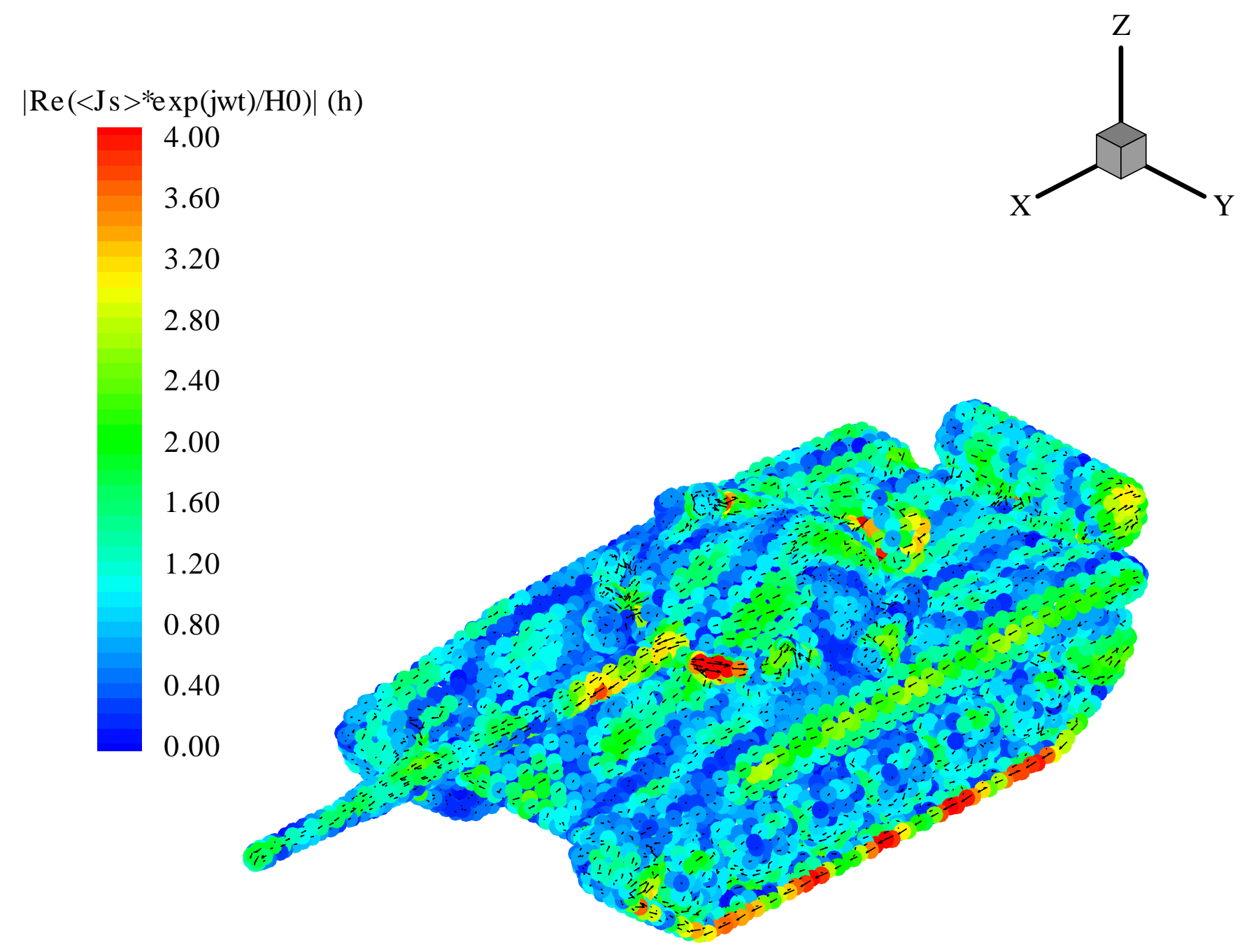

Figure 7(c) 


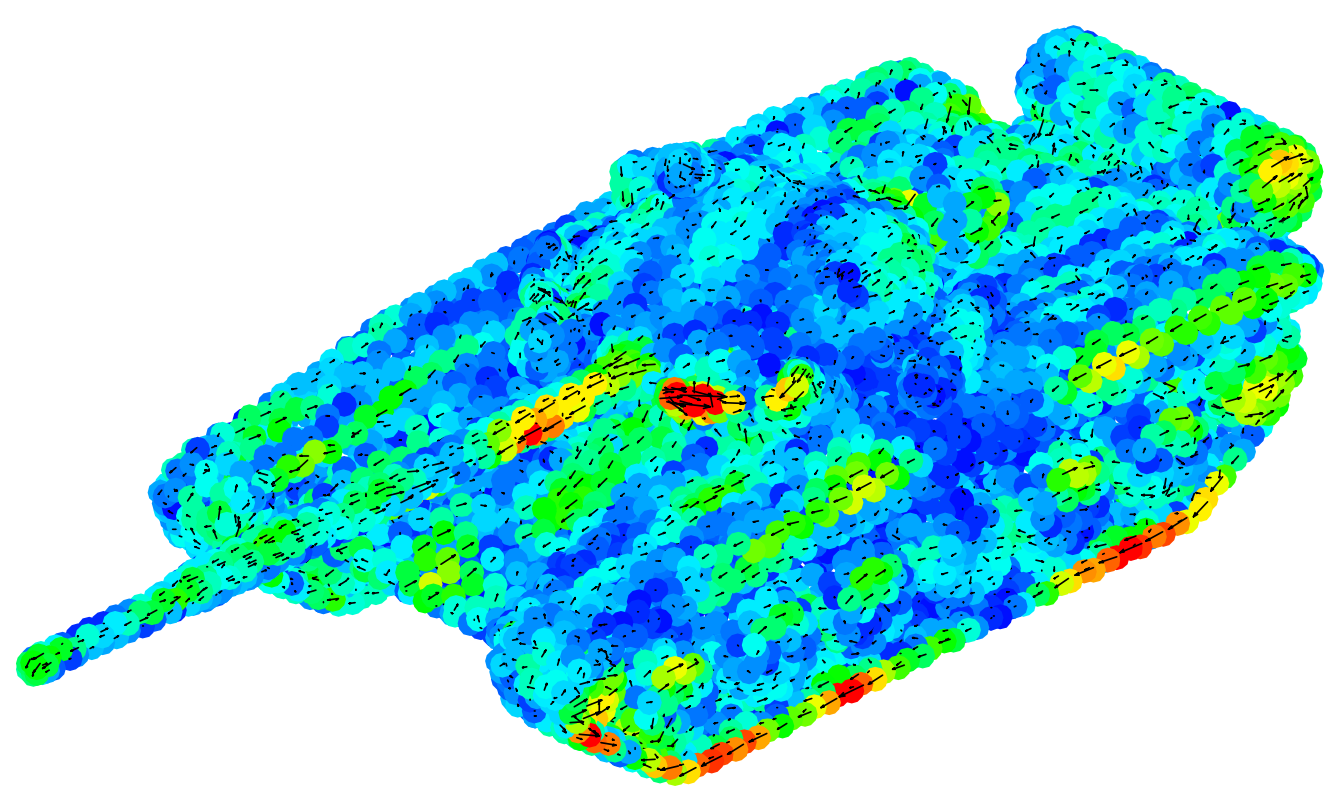

Figure 7(d) 


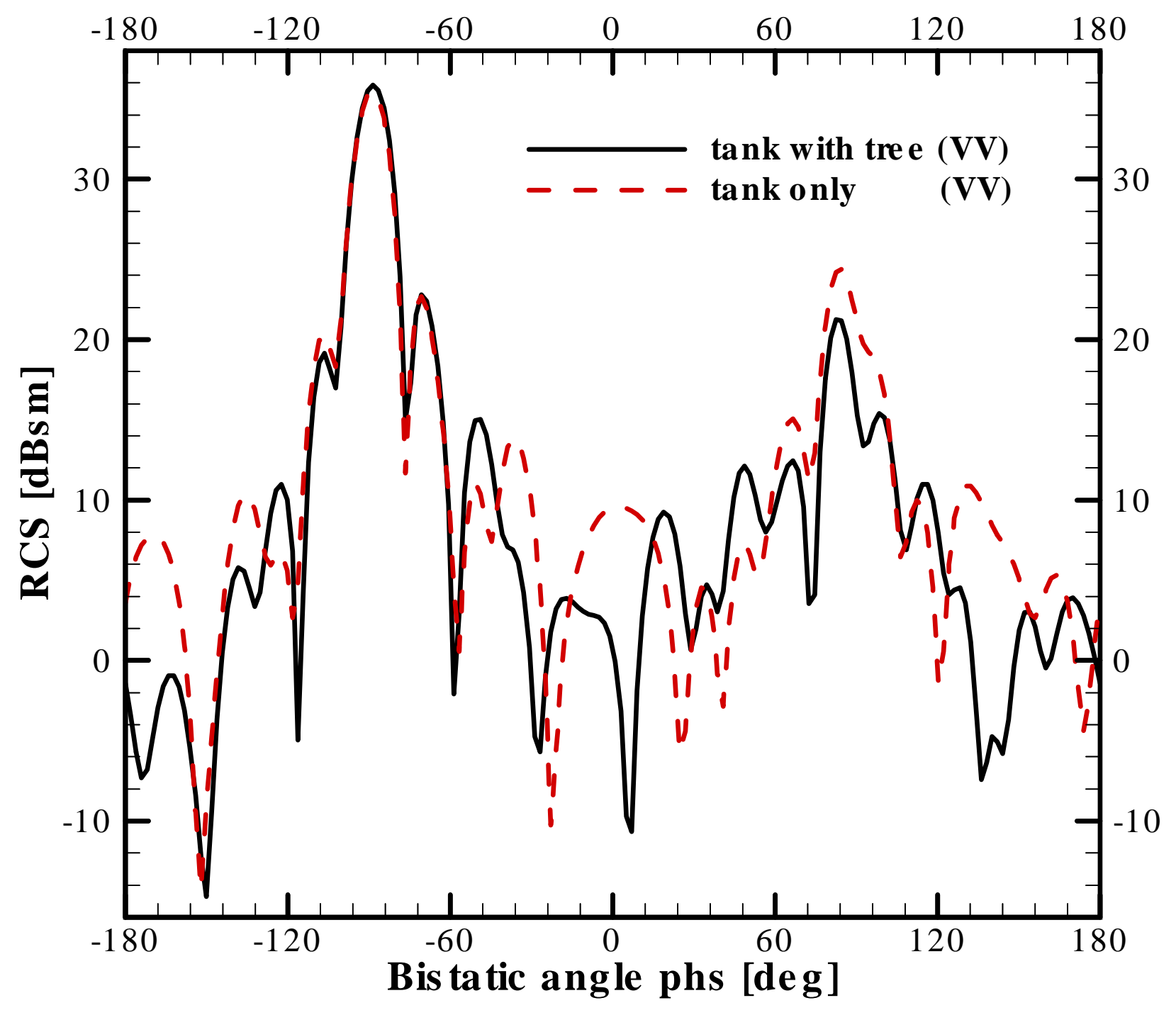

Figure 8(a) 


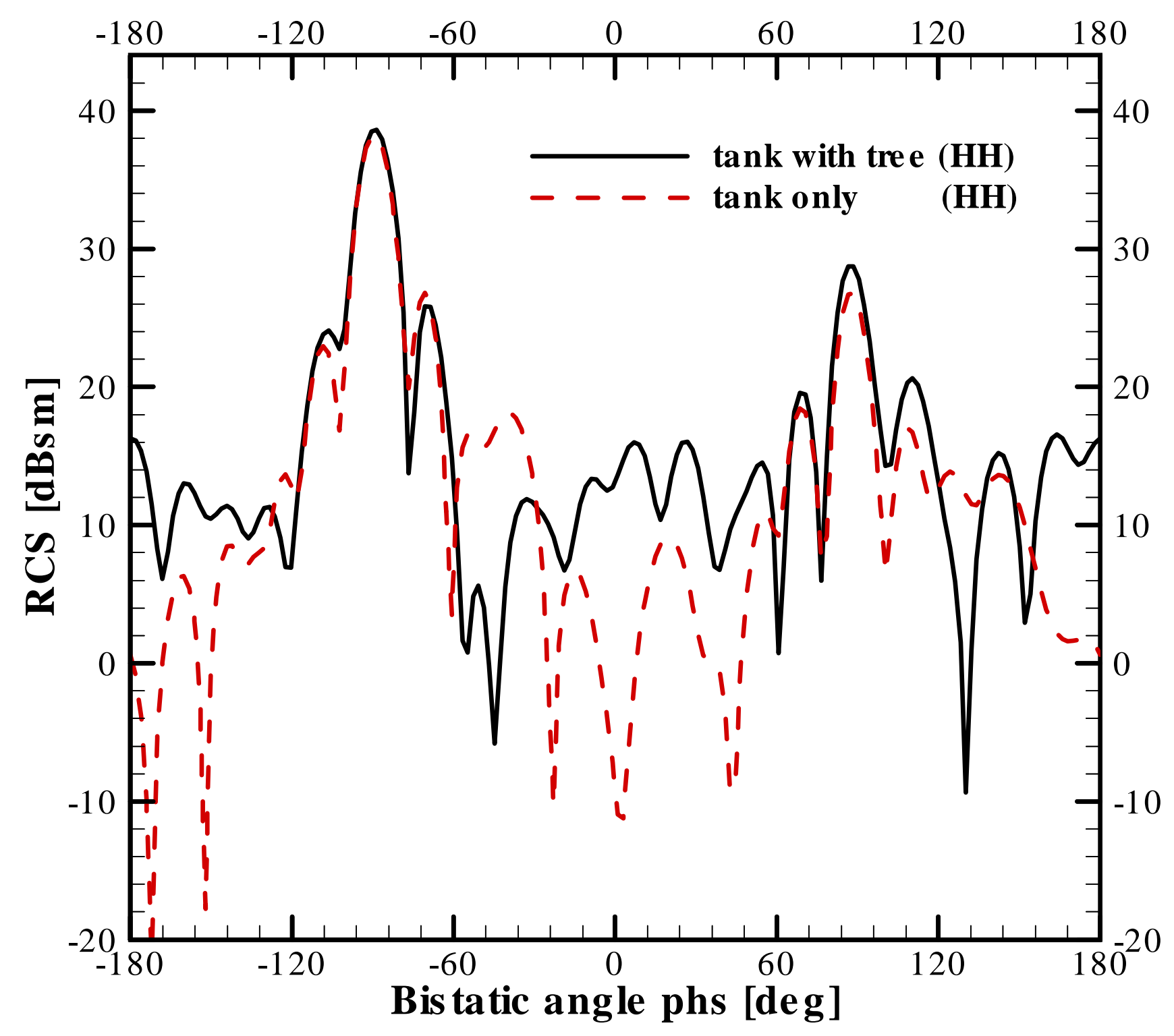

Figure 8(b) 


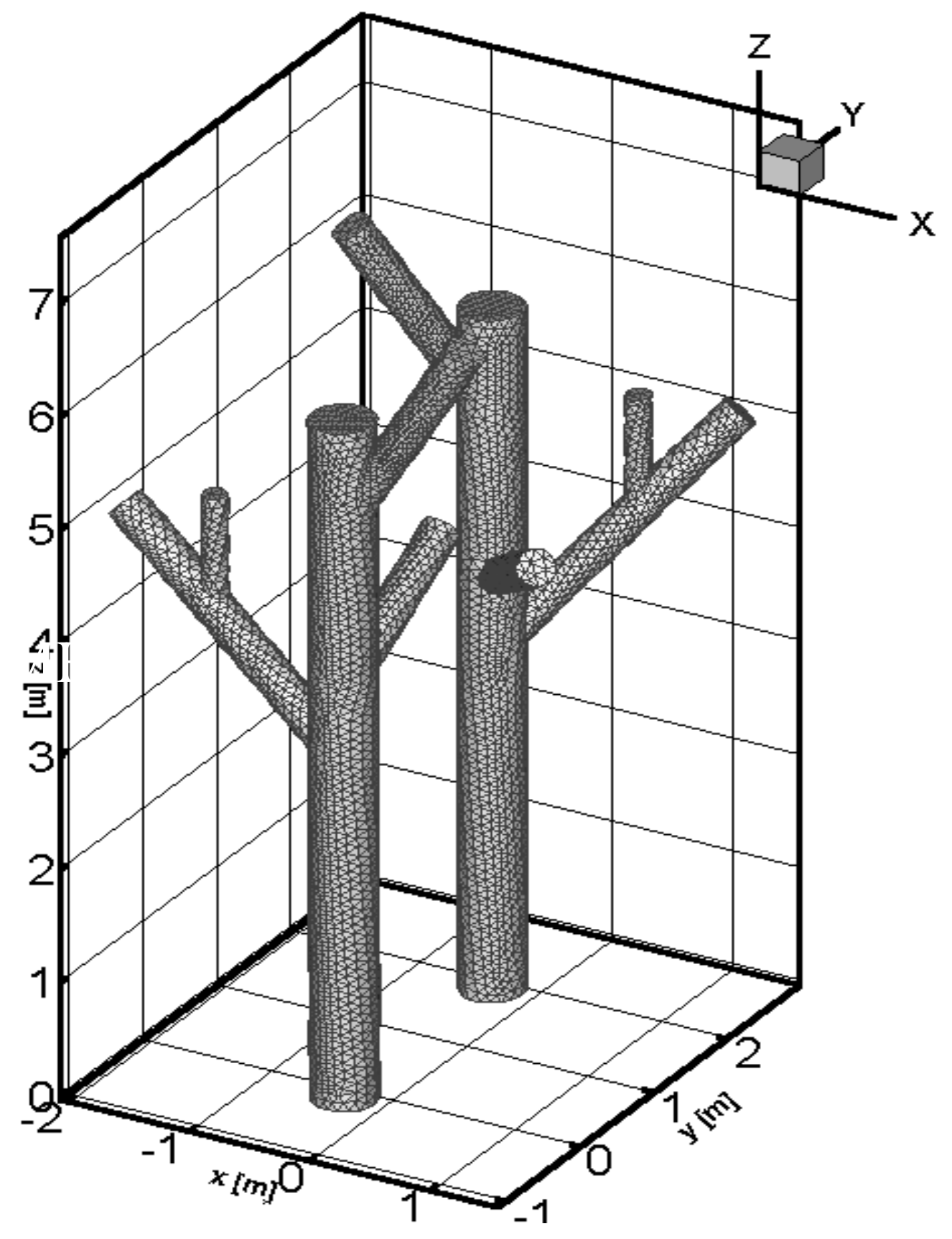

Figure 9 


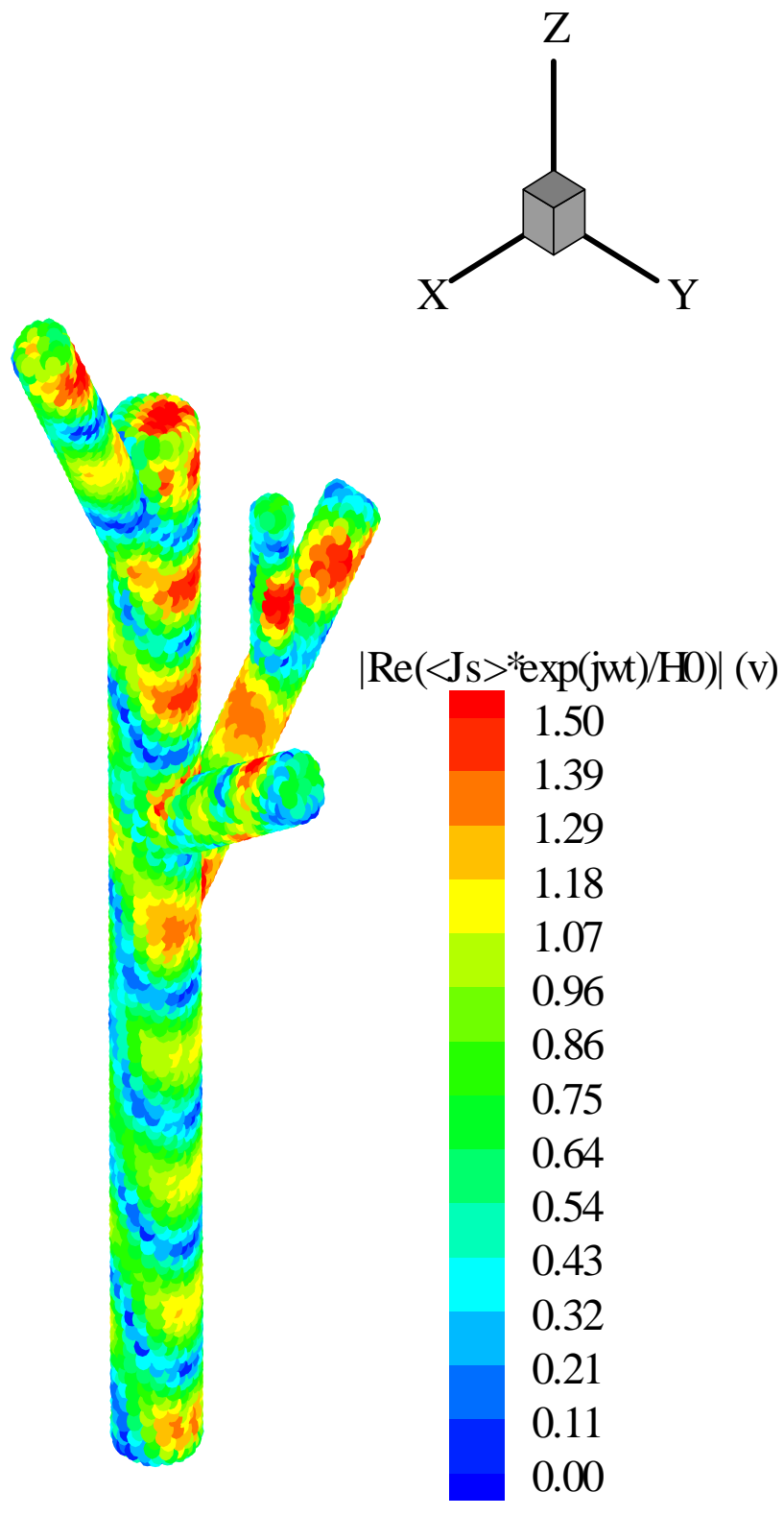

One tree

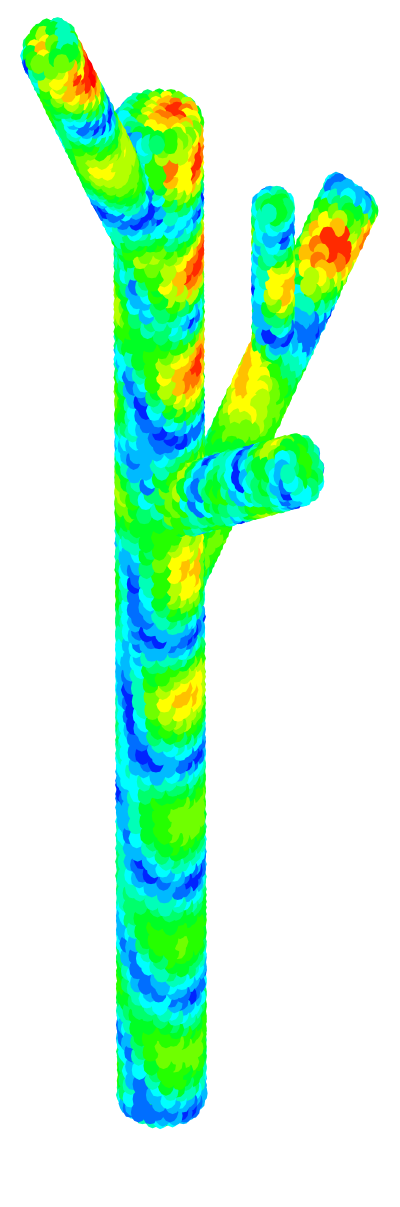

Two trees 


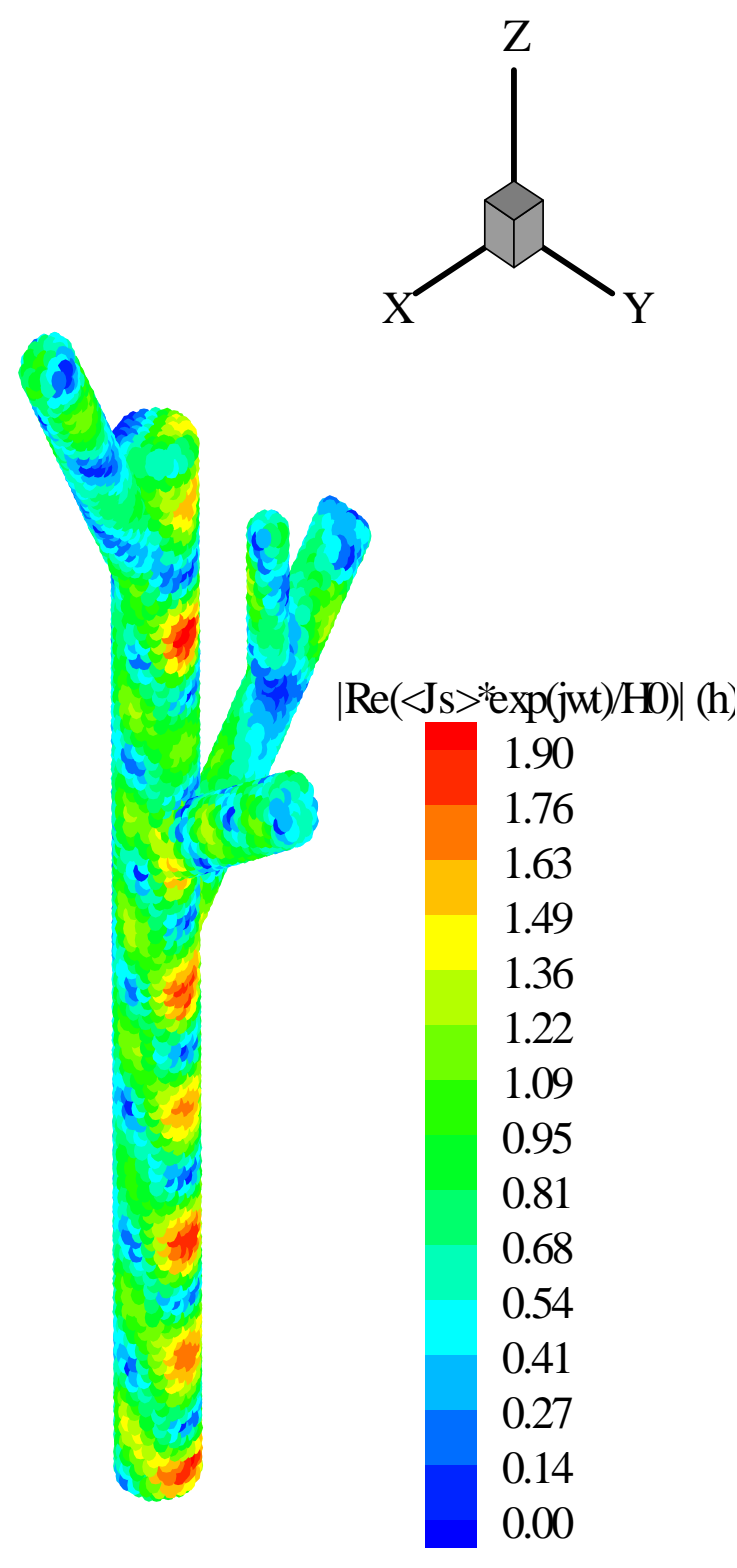

One tree

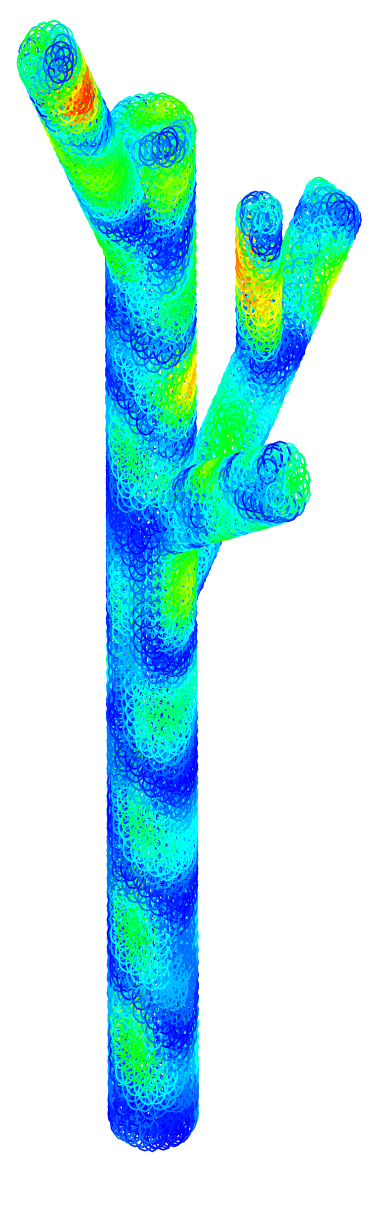

Two trees 


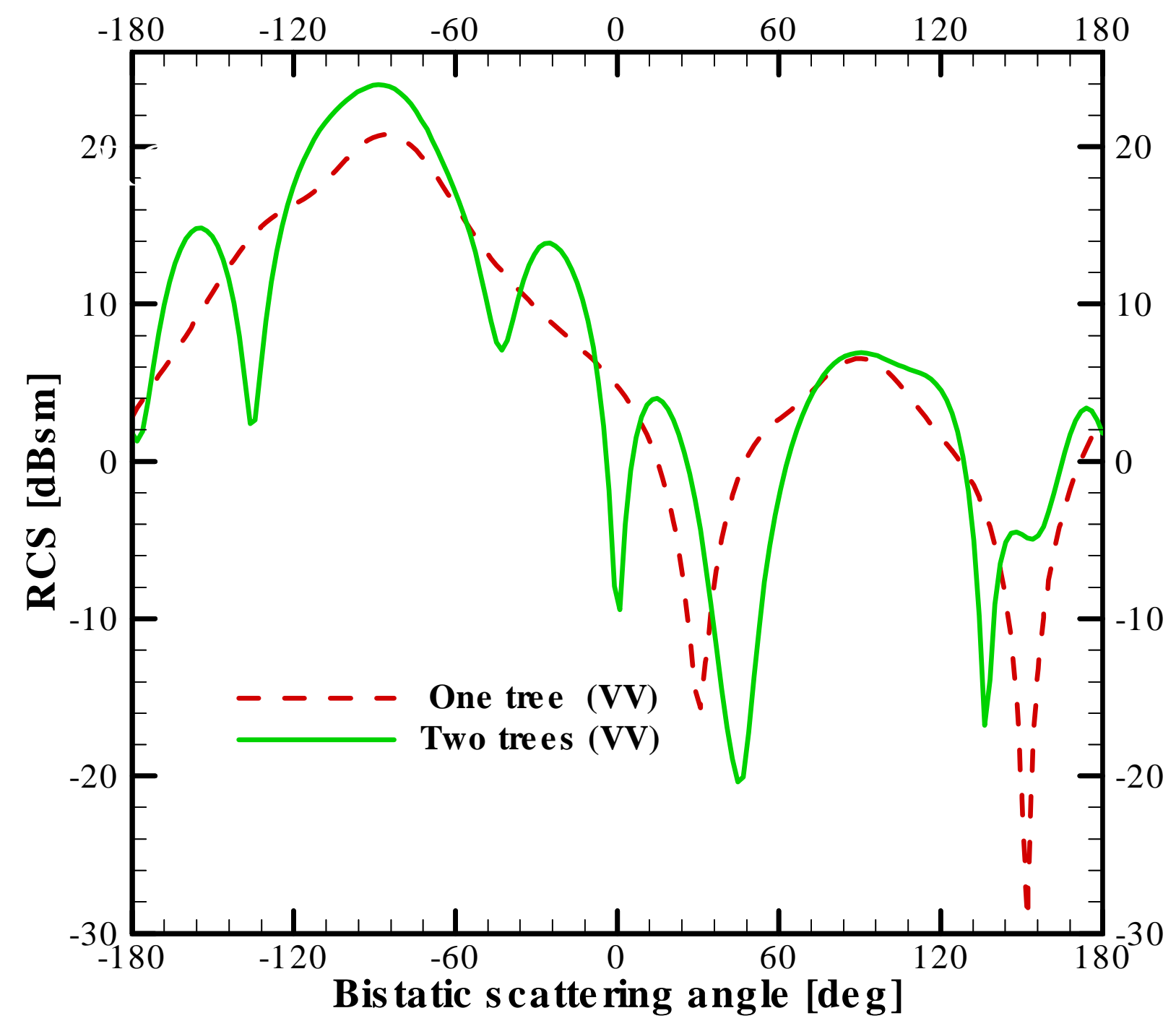

Figure 11(a) 


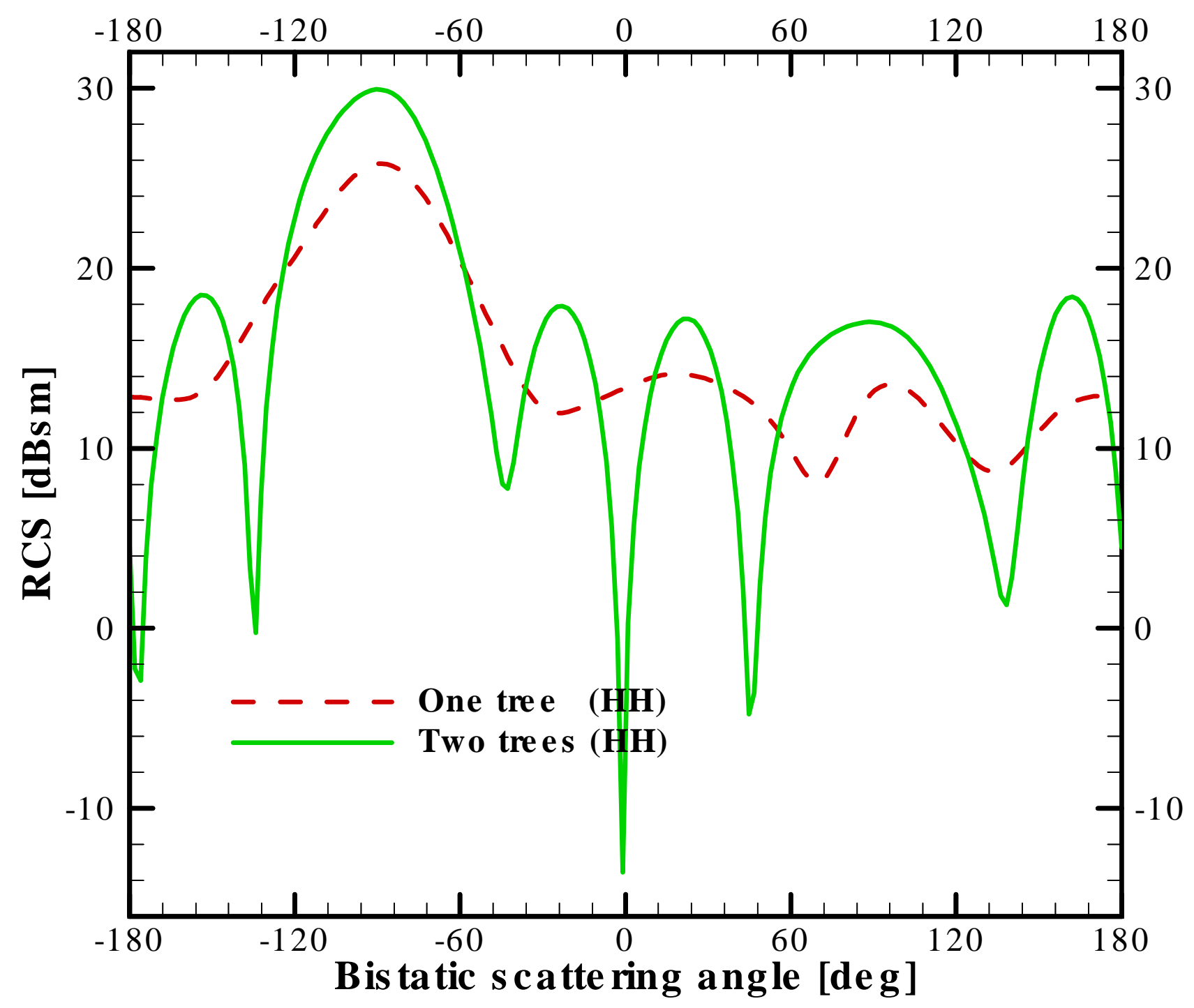

Figure 11(b) 


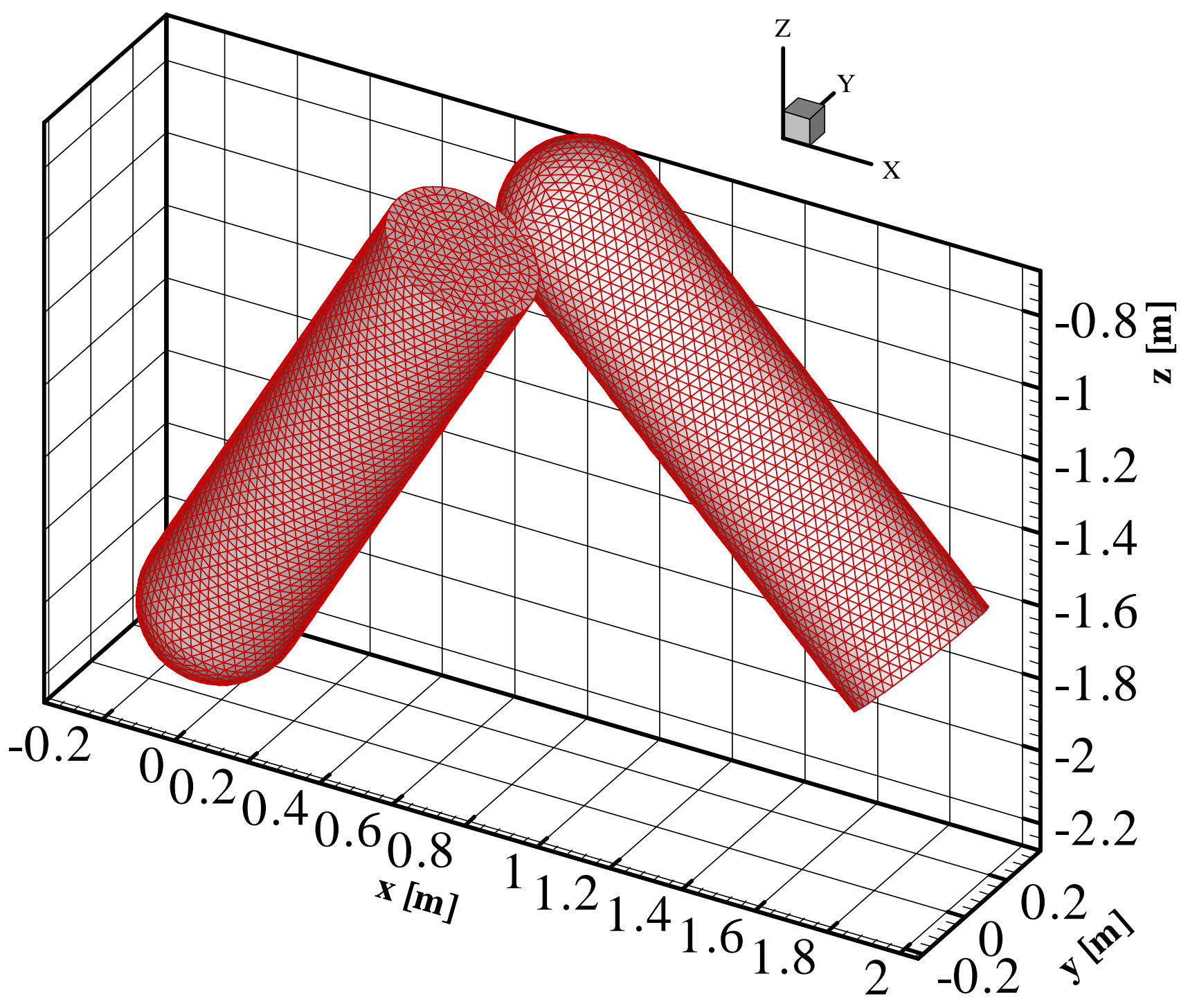

Figure 12 


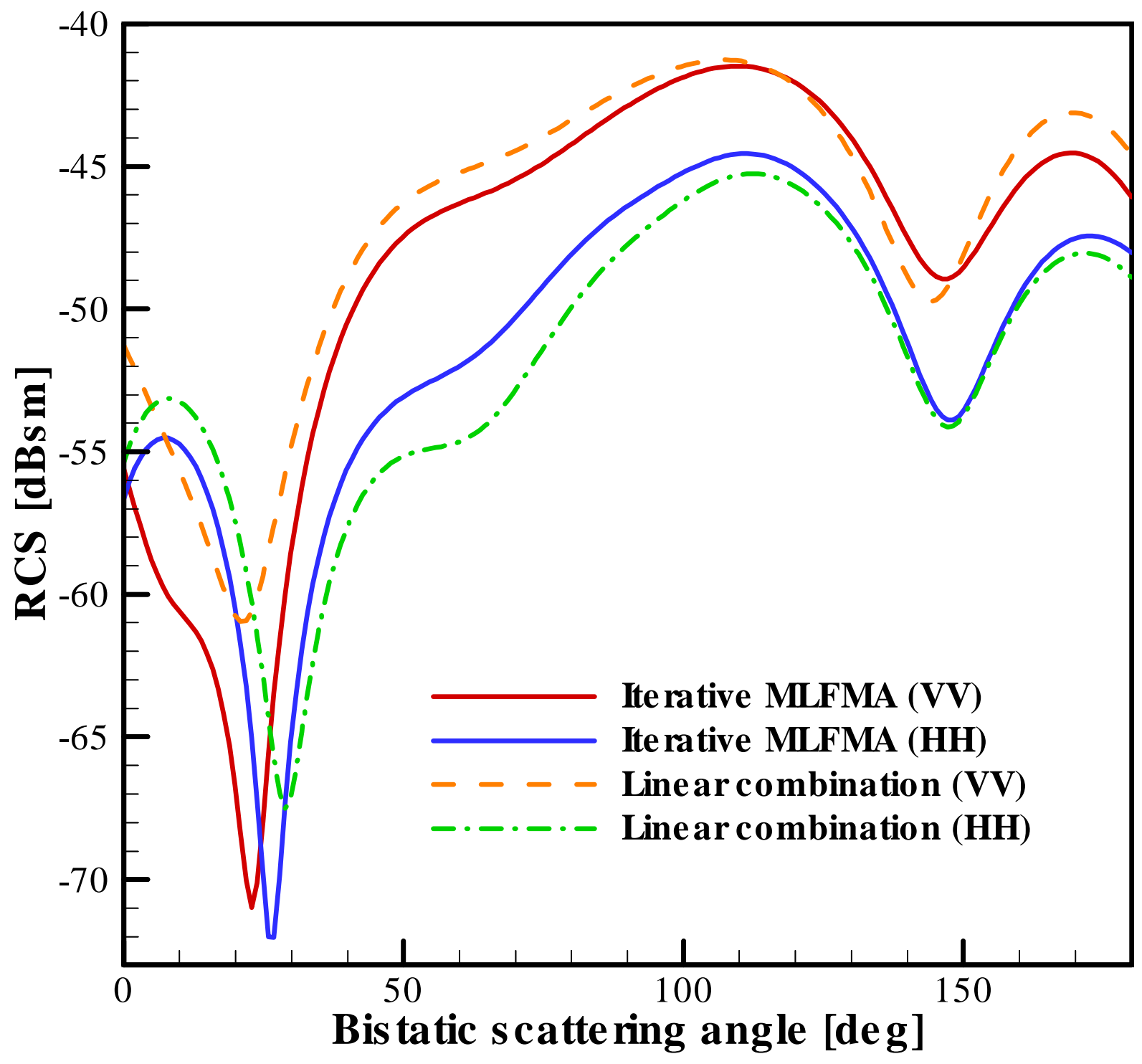

Figure 13 


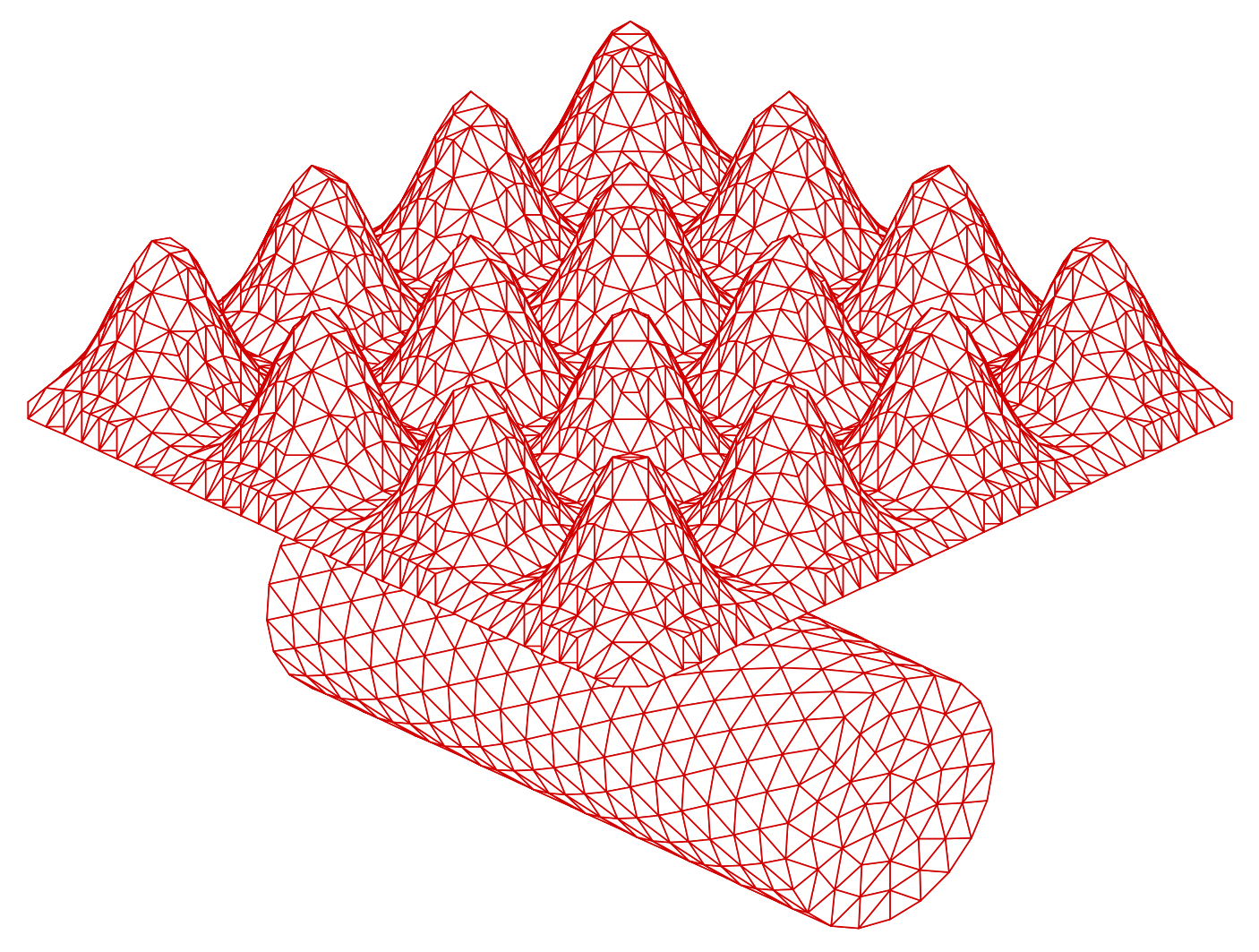

Figure 14 


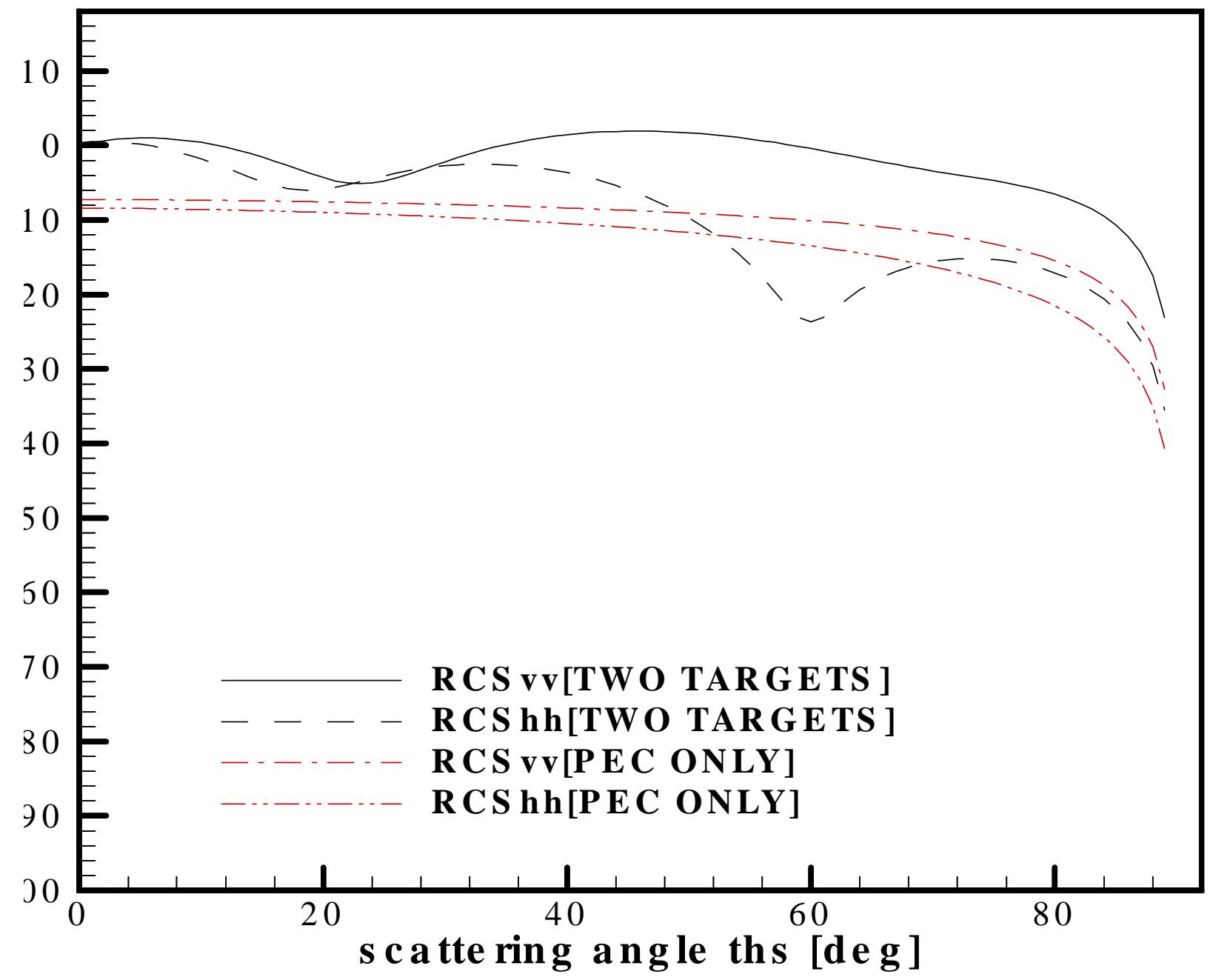

Figure 15(a) 


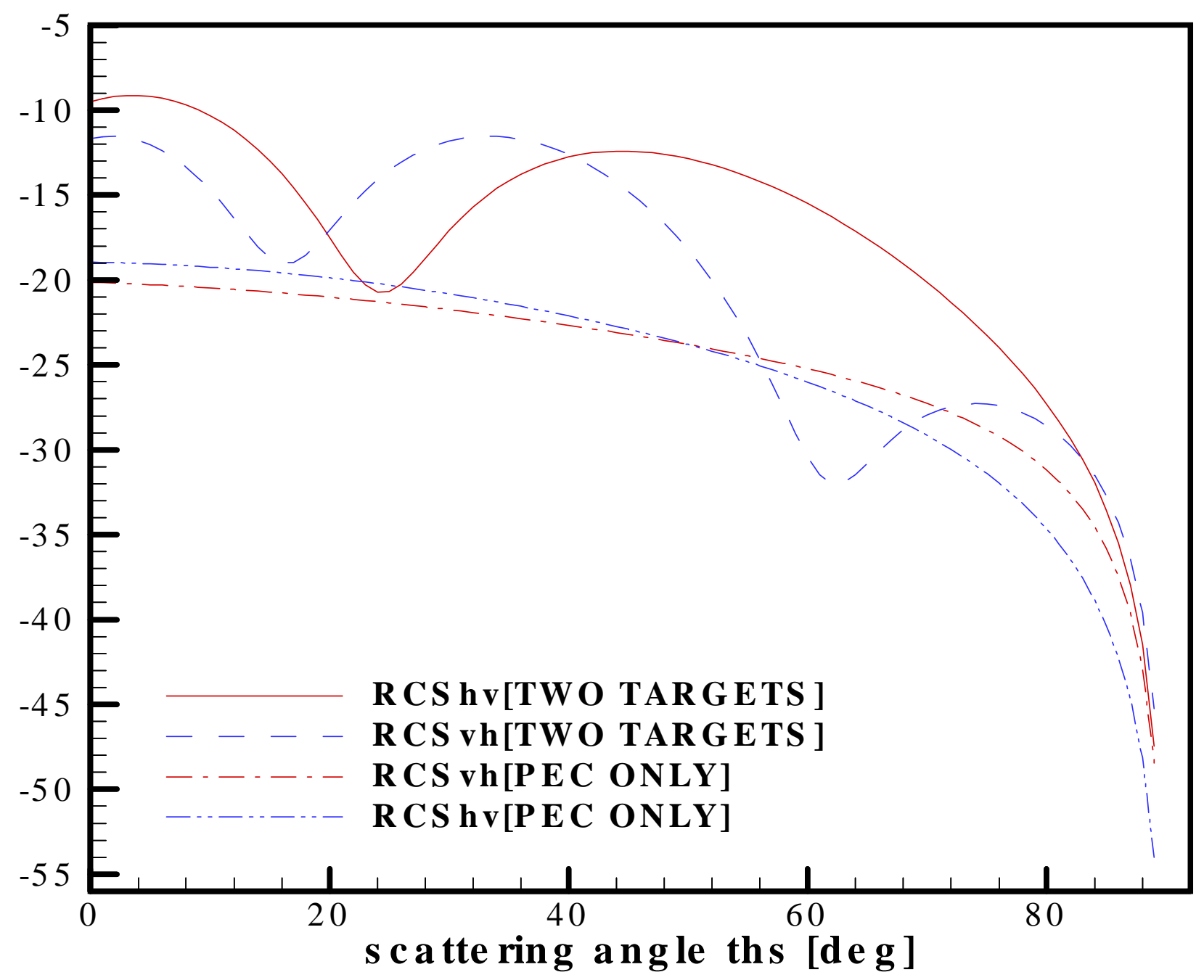

Figure 15(b) 